\title{
Adiposity and cancer risk: new mechanistic insights from epidemiology
}

\author{
Andrew G. Renehan', Marcel Zwahlen² and Matthias Egger ${ }^{2}$ \\ Abstract | Excess body adiposity, commonly expressed as body mass index (BMI), is a risk \\ factor for many common adult cancers. Over the past decade, epidemiological data have \\ shown that adiposity-cancer risk associations are specific for gender, site, geographical \\ population, histological subtype and molecular phenotype. The biological mechanisms \\ underpinning these associations are incompletely understood but need to take account of \\ the specificities observed in epidemiology to better inform future prevention strategies.
}

\begin{abstract}
Body mass index (BMI). An anthropometric measure of body adiposity defined as the body mass (in kilograms) divided by the square of the body height (in metres).

Intermediary biomarkers Biomarkers that predict the development of a disease and thought to be on the causal pathway to the development of that disease.
\end{abstract}

'Institute of Cancer Sciences, Manchester Academic Health Science Centre, The Christie NHS Foundation Trust, University of Manchester Wilmslow Road, Manchester M2O 4BX, UK. IInstitute of Social and Preventive Medicine (ISPM), University of Bern, Finkenhubelweg 11, Bern CH-3012, Switzerland Correspondence to A.G.R. e-mail: andrew.renehan@ics. manchester.ac.uk
A large volume of epidemiological data have established that body mass index (BMI), as an approximation of general adiposity, is a risk factor for several, but not all, common cancers ${ }^{1,2}$. However, there is as yet only indirect evidence that reversal of excess weight (for example, through bariatric surgery) reduces the risk of cancer incidence ${ }^{3}$, and the effect of such interventions may be observable only after a decade. In the meantime, a better understanding of the biological mechanisms underpinning this link might identify intermediary biomarkers of cancer risk and potential new preventive strategies. As the prevalence of overweight and obesity have inexorably risen in developed and less-developed countries in the past three decades, addressing the prevention of cancers attributed to excess adiposity is a globally important health problem ${ }^{4}$.

In their seminal review in 2004, Calle and Kaaks ${ }^{5}$ proposed three 'hormonal' candidate mechanisms for the adiposity-cancer link: altered sex hormone metabolism, increased insulin levels and bioavailability of insulin-like growth factor I (IGF1), and adipokine pathophysiology. In the intervening years, the mechanism of adipokine pathophysiology has been expanded to include systemic (subclinical) inflammation. In this Review, we first provide an update on the epidemiological evidence on the associations between BMI and cancer risk, in an attempt to identify clues about different prevailing mechanisms for the increased risk of cancer according to different sites, gender, geographical populations, histological subtypes and molecular phenotypes. Second, we summarize the aforementioned candidate mechanisms and highlight several shortfalls to these hypotheses. Third, we summarize the effect of weight-losing interventions on intermediary biomarkers from the above candidate systems. Fourth, we describe in more detail how epidemiology has informed the emerging importance of body fat distribution, particularly local ectopic fat, as a paracrine mechanism for cancer development. Finally, we turn to future directions and examine how new technologies may better define body fat compartments at population levels. As a prelude, TABLE 1 summarizes current methods for measuring body adiposity and body fat distribution ${ }^{6}$.

\section{BMI and cancer risk}

Specificity of BMI-cancer associations. The second report of the World Cancer Research Fund and American Institute for Cancer Research (WCRF/AICR) global perspective ${ }^{2}$ in 2007 concluded that there was "convincing" evidence that body fatness, generally measured by calculating BMI, was associated with an increased risk of oesophageal adenocarcinoma and cancers of the pancreas, colorectum, postmenopausal breast, endometrium and kidney, and evidence of a "probable" association of body fatness with increased risk of gallbladder cancer. Simultaneously, we reported a systematic review and dose-response meta-analysis of prospective cohort studies (221 datasets including 281,137 incident cancer diagnoses) quantifying incident relative risk for increases of 20 cancer types, expressed per $5 \mathrm{~kg}$ per $\mathrm{m}^{2}$ increase in $\mathrm{BMI}^{1}$. By using a standardized approach across a large number of cancer types, our analysis demonstrated that BMI-cancer associations are: sex specific (for example, the risk of developing colon cancer is more pronounced for men than for women); site specific (for example, the risk of developing colon cancer is more pronounced than that of rectal cancer); histology specific (for example, an association exists for oesophageal adenocarcinoma but not for oesophageal squamous cell 
Table 1 | Anthropometric measurements of adiposity used in the assessment of disease risk

\begin{tabular}{|c|c|c|c|c|}
\hline Measure & Classifications & Parameters* & Correlations ${ }^{\star \ddagger}$ & Comments \\
\hline 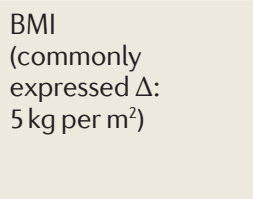 & $\begin{array}{l}\text { - Underweight: }<18.5 \mathrm{~kg} \text { per m } \mathrm{m}^{25} \\
\text { - Normal weight: } 18.5-24.9 \mathrm{~kg} \mathrm{per} \mathrm{m}^{2} \\
\text { - Overweight: } 25.0-29.9 \mathrm{~kg} \mathrm{per} \mathrm{m}^{2} \\
\text { - Obese I: } 30.0-34.9 \mathrm{~kg}_{\text {per m}}^{2} \\
\text { - Obese II: } 35.0-39.9 \mathrm{~kg} \mathrm{per} \mathrm{m}^{2} \\
\text { - Obese III: } \geq 40.0 \mathrm{~kg} \text { per m}{ }^{2}\end{array}$ & $\begin{array}{l}\text { Mean (sd): } \\
\text { - M: } 27.6(4.6) k_{\text {kg per } \mathrm{m}^{2}} \\
\text { - W: } 27.1(5.6) \mathrm{kg} \text { per } \mathrm{m}^{2}\end{array}$ & Height: rho $=0.26$ & $\begin{array}{l}\text { Self-reported results tend to } \\
\text { underestimate BMI in heavier } \\
\text { individuals and with advancing age; } \\
\text { BMI is a poor indicator among the } \\
\text { elderly }\end{array}$ \\
\hline $\begin{array}{l}\text { WC } \\
\text { (commonly } \\
\text { expressed } \Delta: 10 \mathrm{~cm})\end{array}$ & $\begin{array}{l}\mathrm{M}:>102 \mathrm{~cm}^{\S} \\
\mathrm{W}:>88 \mathrm{~cm}^{\S}\end{array}$ & $\begin{array}{l}\text { Mean }(\mathrm{sd}): \\
\text { - M: } 98.8(12.5) \mathrm{cm} \\
\text { W: } 87.8(13.6) \mathrm{cm}\end{array}$ & BMl: rho $=0.46$ & $\begin{array}{l}\text { There are multiple WC measurement } \\
\text { protocols, although these do not } \\
\text { influence associations with outcome }\end{array}$ \\
\hline $\begin{array}{l}\text { WHR } \\
\text { (commonly } \\
\text { expressed } \Delta: 0.1 \text { ) }\end{array}$ & $\begin{array}{l}-\mathrm{M}:>0.90^{\S} \\
\cdot \mathrm{W}:>0.85^{\S}\end{array}$ & $\begin{array}{l}\text { Mean (sd): } \\
\text { - M: } 0.93(0.07) \\
\text { - W: } 0.82(0.07)\end{array}$ & $\begin{array}{l}\text { - BMI: } r \text { ho }=0.33 \\
\text { - WC: } r \text { ho }=0.93\end{array}$ & $\begin{array}{l}\text { WHR better reflects weight changes } \\
\text { in men than in women; similar to } \\
\text { WC, increased WHR predicts for risk } \\
\text { of diabetes and CVD }\end{array}$ \\
\hline $\begin{array}{l}\mathrm{HC} \\
\text { (commonly } \\
\text { expressed } \Delta: 10 \mathrm{~cm})\end{array}$ & None & $\begin{array}{l}\text { Mean (sd): } \\
\text { - M: } 105.6(8.2) \mathrm{cm} \\
\text { - W: } 106.3(11.5) \mathrm{cm}\end{array}$ & $\begin{array}{l}\text { - BMI: rho }=0.47 \\
\text { - WC: rho }=0.90 \\
\text { - WHR: rho }=0.73\end{array}$ & $\begin{array}{l}\text { Increased } \mathrm{HC} \text { predicts for a reduced } \\
\text { risk of CVD }\end{array}$ \\
\hline NAFLD & $\begin{array}{l}\text { Steatosis, NASH, inflammation, injury, } \\
\text { fibrosis and miscellaneous }\end{array}$ & Histology & $\begin{array}{l}\text { MR spectroscopy; } \\
\text { chemical-shift MR }\end{array}$ & Key determinant of insulin resistance \\
\hline
\end{tabular}

BMI, body mass index; CT, computed tomography; CVD, cardiovascular disease; HC, hip circumference; M, men; MR, magnetic resonance; NAFLD, nonalcoholic fatty liver disease; NASH, nonalcoholic steatohepatitis; SAT, subcutaneous adipose tissue; sd, standard deviation; VAT, visceral adipose tissue; W, women; WC, waist circumference; WHR, waist/hip ratio. *Data from Health Survey for England 2006 to 2009. Individual person data aged 20 to 74 years, total: 34,417 (M: 15,553; W: 18,864$)^{6}{ }^{*}$ Spearman correlations with rho values. ${ }^{\text {B }}$ Based on World Health Organization definitions.

\section{Sex hormone}

A family of hormones that

share a basic chemical (steroidal) structure. These hormones include androgens, oestrogens and progesterone, and they have important effects on sexual development and reproductive functions.

Bioavailability

The proportion of a substance that can be used physiologically by target tissues.

Cohort studies

Studies in which a group of individuals is investigated prospectively over time. This is the preferred epidemiological study method for evaluating anthropometric measures and cancer risk

\section{Relative risk}

The risk of cancer (or other disease) in a group of exposed persons divided by the risk in a group of unexposed persons. Relative risk is a commonly used measure of association in epidemiological studies.

Effect modification Also known as effect interaction. When the

association of an exposure with the risk of disease differs in the presence of another exposure. carcinoma (SCC)); and broadly consistent across geographical populations, with some notable exceptions of specificity (for example, there is an increased risk of premenopausal breast cancer in Asia-Pacific populations but not North American and European populations). This analysis also demonstrated that BMI-cancer risk associations exist for a wider range of malignancies than previously thought, including thyroid cancer, malignant melanoma in men, multiple myeloma, leukaemia and non-Hodgkin lymphoma.

Subsequent updated dose-response meta-analyses, using data mainly from prospective studies, reported positive associations between BMI and subsequent increased risk of pancreatic cancer (after taking account of the effect modification of smoking, discussed below) ${ }^{7}$, ovarian cancer (after taking account of the effect modification of hormone replacement therapy (HRT), discussed below) ${ }^{8,9}$ and Hodgkin lymphoma ${ }^{10}$. Single-study reports indicate modest associations between BMI and bladder cancer ${ }^{11}$ and increased BMI in early adulthood and glioma risk ${ }^{12}$; a meta-analysis showed no associations between BMI and testicular cancer ${ }^{13}$.

A recently published large analysis of the UK Clinical Practice Research Dataset, which includes 5.24 million individuals with 166,955 new cancers (of 22 types), corroborated many of the above findings for many cancer types and with similar risk estimates ${ }^{14}$. Importantly, as this was a 'same population' analysis, the authors were able to observe within-population heterogeneity in the effects of BMI on the risk of developing multiple cancer types, and they speculate that "different mechanisms are associated with different cancer sites and different patient subgroups".
When the relationship between BMI and risk of all prostate cancer is examined, risk estimates generally show no association ${ }^{1}$, or even show an inverse association $^{14}$, and there is often considerable between-study heterogeneity . Previously, there had been debate about whether such heterogeneity reflects effect modification due to different levels of prostate-specific antigen (PSA; also known as KLK3) screening across different populations and for different periods of time ${ }^{15}$. In recent years, the relationship between BMI (and indeed, other anthropometric measures) and prostate cancer risk has become clearer. Specifically, there is considerable evidence from prospective cohorts demonstrating that increased BMI is associated with increased risk of advanced-stage prostate cancer ${ }^{16,17}$. TABLE 2 summarizes gender-specific summary risk estimates per $5 \mathrm{~kg}$ per $\mathrm{m}^{2}$ increase in BMI for those cancers for which the evidence for an association is strongest ${ }^{18}$.

Adult weight gain is also associated with incident risk of cancer. A recently reported dose-response metaanalysis of 50 prospective studies demonstrated that patterns of association between adult weight gain and cancer incidence mirror those for BMI and cancer ${ }^{19}$. As adult weight gain correlates with later-adulthood BMI (cohort baseline), and this is the BMI value most commonly determined in epidemiological studies assessing BMI-cancer associations, it is unclear whether adult weight gain is more informative for assessing cancer risk than BMI per se.

Effect modifications of BMI-cancer associations. There are three cancer types for which the associations between BMI and cancer risk are consistently inverse: 
Anthropometric measures Measurements of the size or proportions of the human body: for example, weight, height and waist circumference.

\section{Summary risk estimates}

The weighted summations of collections of study-level risks derived from meta-analyses of studies. These estimates are typically reported as risk or point estimates with $95 \%$ confidence intervals.

\section{Confounding}

The association or lack of association with an exposure that is actually due to another factor that determines the occurrence of a disease but that is also associated with the exposure

\section{Anovulation}

A menstrual cycle that is not accompanied by the discharge of an egg from the ovary. lung cancer, oesophageal SCC, and head and neck cancers. It is now established that cigarette smoking is an effect modifier of the associations between BMI and lung cancer ${ }^{14,20}$ and between BMI and oesophageal $\mathrm{SCC}^{21}$, and it is the most likely explanation for the positive association between leanness and increased risk of head and neck cancers ${ }^{22,23}$. Given that mean BMI values are generally lower in ever smokers than in never smokers ${ }^{24}$, and that smoking is a strong risk factor for lung cancer and oesophageal SCC, the observed inverse associations probably reflect confounding or, in adjusted analyses, residual confounding by smoking ${ }^{25}$. In the absence of smoking, associations between BMI and these cancers are probably close to null. Smoking also affects the association between BMI and pancreatic cancer ${ }^{7}$, but in this case there is a positive association between BMI and cancer risk in the absence of smoking, whereas there is no association in the presence of smoking.

An additional effect modifier of BMI-cancer risk associations is HRT. The risk estimates between BMI and cancer stratified by HRT use are shown in TABLE 3 for postmenopausal breast ${ }^{26}$, endometrial ${ }^{27}$ and ovarian $^{8}$ cancers. In all three cancer types, associations between BMI and cancer risk are strongest for never HRT users, and associations are attenuated among ever users. The type of HRT (oestrogen alone versus combined oestrogen and progesterone) does not seem to be important. This suggests that endogenous sex hormones are on the causal pathway between adiposity and occurrence of these cancers; when these pathways are diluted in the relatively elevated doses of exogenous sex hormones in HRT, BMI-cancer risk associations are less pronounced.
BMI and premenopausal breast cancer. The case of adiposity and premenopausal breast cancer risk deserves specific mention. Data from prospective studies consistently report an inverse association between BMI and premenopausal breast cancer risk (approximately $10 \%$ reduced risk per $5 \mathrm{~kg}$ per $\mathrm{m}^{2}$ ), in North American and European populations ${ }^{1,28}$. Conventionally, this is explained by ovarian-derived androgen excess and chronic anovulation, and an associated reduction of luteal-phase progesterone production (progesterone deficiency). This hypothesis is supported by two analyses of the serum levels of various sex hormones in large prospective cohorts ${ }^{29,30}$, which reported that excess androgen levels were associated with increased premenopausal breast cancer risk.

However, it is incorrect to assume that this association presents a 'protective' effect of female sex hormones. Alternative explanations, including methodological reasons, may exist. Thus, by contrast, among women from Asia-Pacific populations, increased BMI is associated with an increased risk of premenopausal breast cancer (approximately 15\% increased risk per $5 \mathrm{~kg}$ per $\left.\mathrm{m}^{2}\right)^{1,31}$. Additionally, among women from North American and European populations, associations between anthropometric measures of abdominal fatness (such as waist circumference (WC) and waist/hip ratio (WHR)) and premenopausal breast cancer risk are generally null or modestly positive $e^{32-34}$. Collectively, these observations suggest that, in some populations, BMI might be too crude a measure of body fatness to accurately quantify the relationship between adiposity and premenopausal breast cancer.

\begin{tabular}{|c|c|c|c|c|c|}
\hline \multirow[t]{2}{*}{ Cancer type } & \multirow[t]{2}{*}{ Cancer subtype } & \multirow{2}{*}{$\begin{array}{l}\text { Number of } \\
\text { cohorts }\end{array}$} & \multicolumn{2}{|c|}{ Summary risk estimate $(95 \% \mathrm{Cl})$} & \multirow[t]{2}{*}{ Refs } \\
\hline & & & Men & Women & \\
\hline Colon & Adenocarcinoma & 29 & $1.24(1.20,1.28)$ & $1.09(1.05,1.13)$ & 1 \\
\hline Rectum & Adenocarcinoma & 29 & $1.09(1.06,1.12)$ & $1.02(1.00,1.05)$ & 1 \\
\hline Oesophageal & Adenocarcinoma & 5 & $1.52(1.33,1.74)$ & $1.51(1.31,1.74)$ & 1 \\
\hline Oesophageal & SCC & 5 & $0.71(0.60,0.85)$ & $0.57(0.47,0.69)$ & 1 \\
\hline Gastric & Adenocarcinoma & 8 & $0.97(0.88,1.06)$ & $1.04(0.90,1.20)$ & 1 \\
\hline Liver & $\mathrm{HCC}$ & 9 & $1.19(1.09,1.29)$ & $1.12(1.03,1.22)$ & 18 \\
\hline Gallbladder & Adenocarcinoma & 4 & $1.09(0.99,1.21)$ & $1.59(1.02,2.47)$ & 1 \\
\hline Pancreatic & Adenocarcinoma & 23 & $1.13(1.04,1.22)$ & $1.10(1.04,1.16)$ & 7 \\
\hline Lung & Not investigated & 13 & $0.76(0.70,0.83)$ & $0.80(0.66,0.97)$ & 1 \\
\hline Kidney & Not investigated & 12 & $1.24(1.15,1.34)$ & $1.34(1.25,1.43)$ & 1 \\
\hline $\begin{array}{l}\text { Advanced-stage } \\
\text { prostate }^{\star}\end{array}$ & Not investigated & 23 & $1.08(1.04,1.12)$ & NA & 17 \\
\hline Postmenopausal breast & Not investigated & 34 & NA & $1.12(1.08,1.16)$ & 1 \\
\hline Premenopausal breast & Not investigated & 34 & NA & $0.92(0.88,0.97)$ & 1 \\
\hline Endometrial & Not investigated & 19 & NA & $1.59(1.50,1.68)$ & 1 \\
\hline Ovarian & Not investigated & 34 & NA & $1.06(1.00,1.12)$ & 9 \\
\hline
\end{tabular}

BMI, body mass index; $\mathrm{Cl}$, confidence interval; HCC, hepatocellular carcinoma; NA, not applicable; SCC, squamous cell carcinoma. *Defined differently across studies but including: American Joint Committee on Cancer stages 3 and 4; metastatic cancer; Whitmore-Jewett stages $C$ and $D$; high grade; and Gleason grade $\geq 7$. 
Table 3 | Summary risk estimates for associations between BMI and risk of selected female cancers by HRT

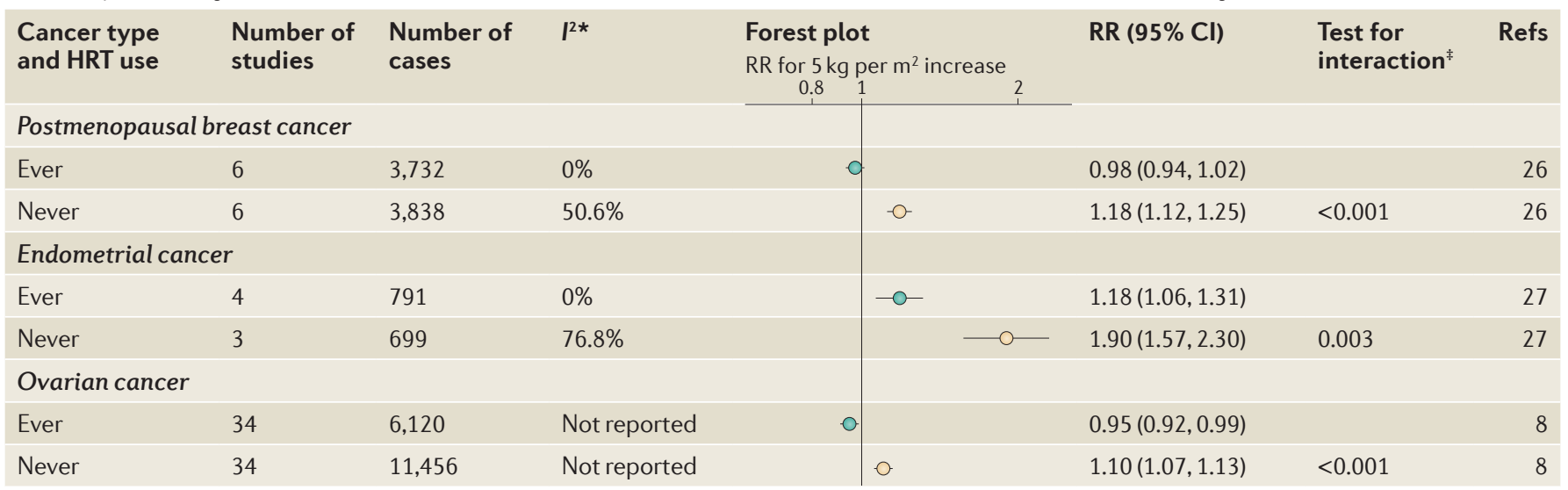

BMI, body mass index; $\mathrm{Cl}$, confidence interval; HRT, hormone replacement therapy; RR, relative risk. ${ }^{*}{ }^{2}$ is a statistic used as an indicator of between-study heterogeneity.

‘Test for interaction using meta-regression.

Another alternative explanation is that height squared in the BMI formula inadequately adjusts for height in women ${ }^{35}$ (height is an independent risk factor for breast cancer $^{36}$ ). For example, short, obese young women may have a low risk of premenopausal breast cancer owing to their low height, even though they have high $\mathrm{BMI}^{37}$.

Associations with molecular phenotype. Increasingly, tumours are classified based on their molecular profile. Such classifications afford opportunities to enhance the exploration of exposure-disease associations and to infer specific causal pathways. Large epidemiological studies are now exploring these relationships and their interaction with BMI (summarized in TABLE 4).

For breast cancer, a relatively simple molecular classification is based on immunohistochemical expression of oestrogen receptor (ER) and progesterone receptor (PR). The evidence linking BMI and ER-PR status with breast cancer risk has been mounting and was initially summarized in a meta-analysis published in 2009 ( 9 cohorts; 22 case-control studies $)^{38}$. Since then, additional findings from several large cohorts have been published ${ }^{39-44}$, as has a pooled analysis from the Breast Cancer Association Consortium ${ }^{45}$. $\mathrm{ER}^{+} \mathrm{PR}^{+}$tumours account for approximately $60 \%$ of premenopausal breast cancers. For these tumours, the BMI-cancer risk associations broadly mirror those seen in studies in which there are no molecular classifications: namely, an approximate $10 \%$ risk reduction for premenopausal breast cancer and 33\% risk increase for postmenopausal breast cancer per $5 \mathrm{~kg}$ per $\mathrm{m}^{2} \mathrm{BMI}$ increment ${ }^{38}$.

For $\mathrm{ER}^{-} \mathrm{PR}^{-}$breast cancer risk, results have been mixed in postmenopausal women, but most cohort studies find no association ${ }^{39,46-49}$ or a reduced risk with increased $\mathrm{BMI}^{43}$. However, two recent studies found positive associations between increasing BMI and the incidence of triple-negative tumours (that is, tumours that are $\mathrm{ER}^{-} \mathrm{PR}^{-}$and do not express human epidermal growth factor receptor 2 (HER2; also known as ERBB2)) in all study participants ${ }^{44}$ and in a subgroup of never HRT users $^{42}$. Among premenopausal women, there may also be positive associations between abdominal adiposity and triple-negative breast cancer ${ }^{41}$. These observations suggest that, in terms of adiposity-driven mechanisms of tumour development, $\mathrm{ER}^{+}$and $\mathrm{ER}^{-}$tumour types may share common pathways. It has been hypothesized that a large proportion of $\mathrm{ER}^{-}$tumours arise from oestrogen-responsive precursor tumours or cells and that oestrogen sensitivity is lost during tumour development ${ }^{50}$. In addition, mammary stem cells are known to be responsive to sex hormones despite not having detectable expression of ER or PR ${ }^{51}$.

Endometrial cancers have long been subclassified into Bokhman's type 1 endometrioid tumours $(\geq 70 \%)$ and type 2 (other histology) tumours ${ }^{52}$; type 1 tumours are oestrogen-driven and conventionally associated with adiposity-driven pathways. The exact definitions of type 2 histological subtypes vary across the literature, but despite this we and others, through nonlinear piecewise meta-analysis ${ }^{27}$ and consortium analyses ${ }^{53}$, respectively, have shown that BMI-cancer risk associations are greater for type 1 than for type 2 endometrial carcinomas.

Histological classification of ovarian cancers is complex and not standardized across epidemiological studies. Nonetheless, differential associations exist between subtypes of ovarian cancer and BMI. A pooled analysis of studies participating in the Ovarian Cancer Association Consortium (15 case-control studies) convincingly showed that there are no associations between BMI and the most common type of ovarian cancer, serous ovarian carcinoma (which accounts for $66 \%$ of ovarian cancer cases), whereas positive associations exist with lesscommon histological types, such as mucinous ovarian carcinomas and endometrioid carcinomas of the ovary ${ }^{54}$. These findings are consistent with cohort studies in the Collaborative Group on Epidemiological Studies of Ovarian Cancer (17 cohorts) ${ }^{8}$.

There are at least two main classes of colorectal cancer: those exhibiting microsatellite stability (MSS; 85\% of cases) and those with microsatellite instability (MSI; 15\% of cases). Data from three case-control studies suggest that positive associations between BMI and colon cancer are restricted to MSS tumours ${ }^{55-57}$. By contrast, pooled data from The Netherlands Cohort Study and Melbourne 
Attributable risk

In epidemiology, this is the difference in the rate of a

condition between an

exposed population and

an unexposed population

Peripheral adipose tissue Fat stores other than intra-abdominal fat (mainly subcutaneous fat).
Collaborative Cohort Study found no effect modification for colorectal cancer by MSI status and BRAF mutation status $^{58}$. Differences may reflect lack of a universal definition for CpG island methylator phenotype (CIMP), which is a key determinant of microsatellite status. Additionally, case-control studies in colon cancer indicate that tumour expression of fatty acid synthase (FASN; a regulator of fatty acid-derived energy metabolism) and $\beta$-catenin (a mediator of the WNT pathway encoded by CTNNB1) may modify the effect of BMI on colon cancer risk. Thus, the association between BMI and increased colon cancer risk is limited to colon tumours expressing FASN $^{59}$ or those not expressing $\beta$-catenin ${ }^{60}$.

Taken together, and assuming that these associations are causal (discussed below), a recent study using the GLOBOCAN 2012 data estimated attributable risk and numbers of new cancer cases attributable to high $\mathrm{BMI}^{4}$. These analyses took account of the effect modifications of country-level smoking prevalence and HRT use. The summary estimate reported that $3.6 \%$ of all new cancers in adults aged 30 years and older (excluding non-melanoma skin cancer) in 2012, or $12.8 \%$ of high-BMI-associated cancers, are attributable to high $\mathrm{BMI}^{4}$. These figures are equivalent to an estimated 481,000 new cancers worldwide that might have been caused by high BMI.

Mechanisms that explain adiposity-cancer links need to account for the specificities of these associations for gender, site, histological and molecular subtypes; using observations such as effect modification may also help researchers to narrow down the specific mechanisms underlying these associations in certain cancer types.

\section{Current hypotheses of biological mechanisms}

Three hormonal mechanisms have been proposed to explain links between adiposity and increased cancer risk (FIG. 1): sex hormone metabolism, insulin and IGF signalling, and adipokine pathophysiology $y^{5,61-63}$. Subclinical inflammation has also emerged as important and is intimately connected to the adipokine system.

Sex hormone hypothesis. This hypothesis applies predominantly, but not exclusively, to postmenopausal breast, endometrial and ovarian cancers. In the setting of excess adiposity, increased risk in these cancers is explained by the higher rates of conversion of androgenic precursors to oestradiol through increased aromatase enzyme activity in peripheral adipose tissue - a process known as aromatization ${ }^{64}$. For breast cancer, there is abundant experimental evidence from in vitro and animal models that oestrogens are mitogenic and, indeed, mutagenic: they induce direct or indirect free-radical-mediated DNA damage, genetic instability and mutations in cells in normal and neoplastic mammary tissues ${ }^{65}$. Altered concentrations of circulating oestrogen-related hormones are linked to breast

\section{Table 4 | Molecular epidemiology: associations between BMI and different molecular subtypes in prospective cohort studies}

\begin{tabular}{|c|c|c|c|c|c|c|}
\hline Cancer type & Studies & $\begin{array}{l}\text { Molecular } \\
\text { marker }\end{array}$ & $\begin{array}{l}\text { Percentage of } \\
\text { each cancer } \\
\text { type in study }\end{array}$ & $\begin{array}{l}\text { Risk estimate per } \\
5 \mathrm{~kg} \text { per } \mathrm{m}^{2} \mathrm{BMI} \\
(95 \% \mathrm{Cl})\end{array}$ & Comments & Refs \\
\hline \multirow{2}{*}{$\begin{array}{l}\text { Premenopausal } \\
\text { breast cancer }\end{array}$} & \multirow{2}{*}{$\begin{array}{l}\text { BCAC* }^{\star} \\
\text { meta-analysis } \\
\text { plus updated } \\
\text { studies }\end{array}$} & $\mathrm{ER}^{+} \mathrm{PR}^{+}$ & $\sim 60^{\star}$ & $\sim 10 \%$ risk reduction & \multirow[t]{2}{*}{-} & \multirow[t]{2}{*}{$39-45$} \\
\hline & & $\begin{array}{l}\mathrm{ER}^{-} \mathrm{PR}^{-} \text {and } \\
\text { triple negative }\end{array}$ & $\sim 30^{*}$ & $\begin{array}{l}\sim 80 \% \text { risk increase (with } \\
\text { abdominal adiposity) }\end{array}$ & & \\
\hline \multirow{2}{*}{$\begin{array}{l}\text { Postmenopausal } \\
\text { breast cancer }\end{array}$} & \multirow{2}{*}{$\begin{array}{l}\text { BCAC*, }^{*} \\
\text { meta-analysis } \\
\text { plus updated } \\
\text { studies }\end{array}$} & $\mathrm{ER}^{+} \mathrm{PR}^{+}$ & $\sim 60^{\star}$ & $\sim 33 \%$ risk increase & \multirow[t]{2}{*}{-} & \multirow[t]{2}{*}{$39-45$} \\
\hline & & $\mathrm{ER}^{+} \mathrm{PR}^{-}$ & $\sim 15^{\star}$ & Null association & & \\
\hline \multirow[t]{4}{*}{ Colon cancer } & \multirow[t]{2}{*}{ CCFR } & MSI & $\sim 15$ & $1.05(0.84,1.31)$ & \multirow[t]{2}{*}{-} & \multirow[t]{2}{*}{55} \\
\hline & & MSS & $\sim 85$ & $\begin{array}{l}1.38(1.24,1.54) \\
P_{\text {interaction }}=0.08\end{array}$ & & \\
\hline & \multirow[t]{2}{*}{ HPFS and NHS } & $\beta$-catenin ${ }^{-}$ & 54 & $1.84(1.34,2.53)$ & \multirow{2}{*}{$\begin{array}{l}\beta \text {-catenin is a major mediator } \\
\text { of the WNT pathway and, when } \\
\text { overexpressed, contributes to tumour } \\
\text { progression }\end{array}$} & \multirow[t]{2}{*}{60} \\
\hline & & $\beta$-catenin ${ }^{+}$ & 46 & $\begin{array}{l}1.29(0.83,1.71) \\
P_{\text {interaction }}=0.027\end{array}$ & & \\
\hline \multirow[t]{2}{*}{$\begin{array}{l}\text { Endometrial } \\
\text { cancer }\end{array}$} & \multirow[t]{2}{*}{$\begin{array}{l}\text { Piecewise } \\
\text { meta-analysis }\end{array}$} & Type I & 87 & $2.39(1.90,3.03)^{\varsigma}$ & $\begin{array}{l}\text { Endometrioid (mucinous) } \\
\text { adenocarcinoma }\end{array}$ & \multirow[t]{2}{*}{27} \\
\hline & & Type II & 13 & $1.92(1.50,2.47)^{\varsigma}$ & $\begin{array}{l}\text { Papillary, clear cell, serous and other } \\
\text { carcinomas }\end{array}$ & \\
\hline
\end{tabular}

BCAC, Breast Cancer Association Consortium; BMI, body mass index; CCFR, Colon Cancer Family Registry; Cl, confidence interval; ER, oestrogen receptor; FASN, fatty acid synthase; HPFS, Health Professionals Follow-up Study; MSI, microsatellite instability; MSS, microsatellite stability; NHS, Nurses' Health Study; PR, progesterone receptor. *Percentages of tumour subtypes taken from the BCAC analysis ${ }^{45}$. ${ }^{\ddagger}$ Model as per $10 \mathrm{~cm}$ increment of waist circumference ${ }^{41}$. ${ }^{\S}$ Risk estimates in the piecewise model for $\mathrm{BMI}=32 \mathrm{~kg}$ per $\mathrm{m}^{2}$. 
cancer risk. Specifically, the Endogenous Hormones and Breast Cancer Collaborative Group (EHBCCG) ${ }^{66}$ and the European Prospective Investigation into Cancer and Nutrition (EPIC) study ${ }^{67}$ reported similar findings: postmenopausal breast cancer risk is increased (typically twofold for upper versus lowest quintiles) among women with higher concentrations of circulating sex hormones including dehydroepiandrosterone (DHEA), dehydroepiandrosterone sulfate (DHEAS), $\triangle 4$-androstenedione, testosterone, oestrone and total oestradiol, and decreased concentrations of sex hormone-binding globulin (SHBG). In the EHBCCG

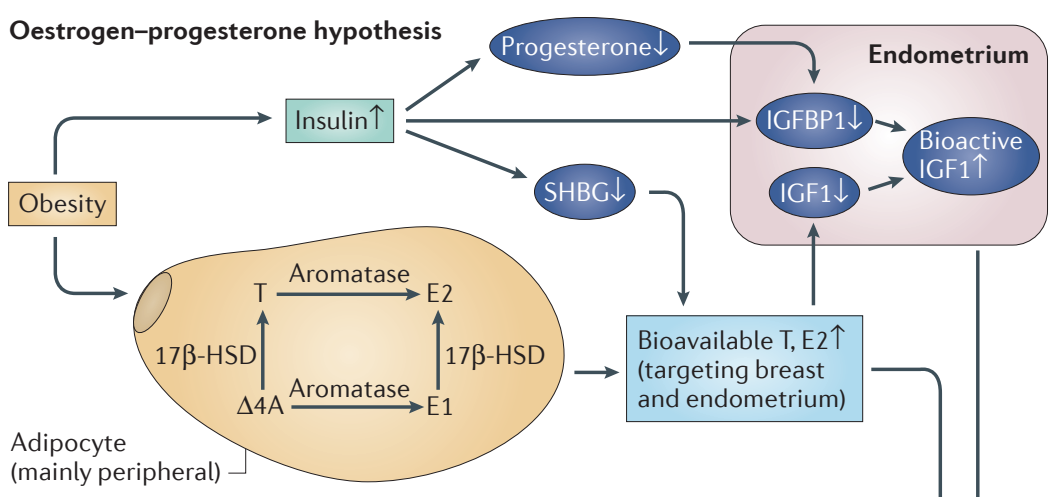

Insulin-IGF hypothesis

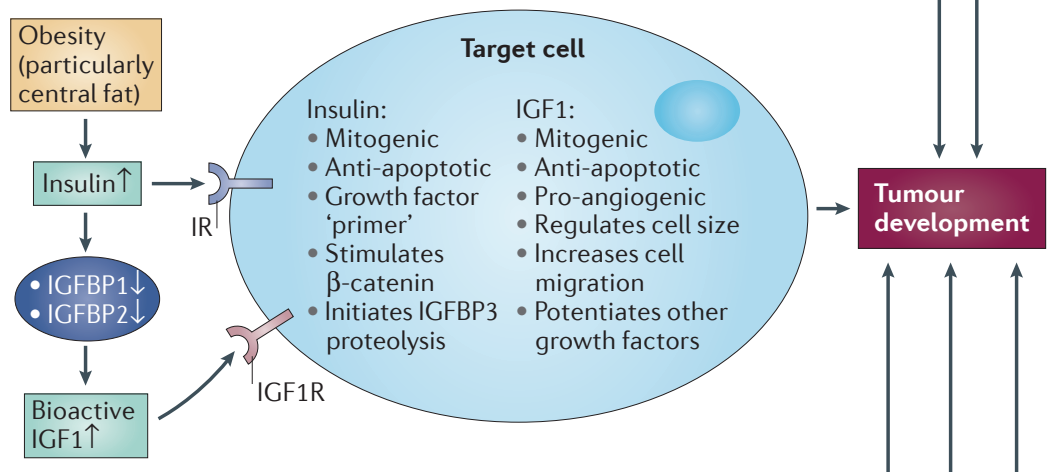

Inflammatory mediators and adipokine hypothesis

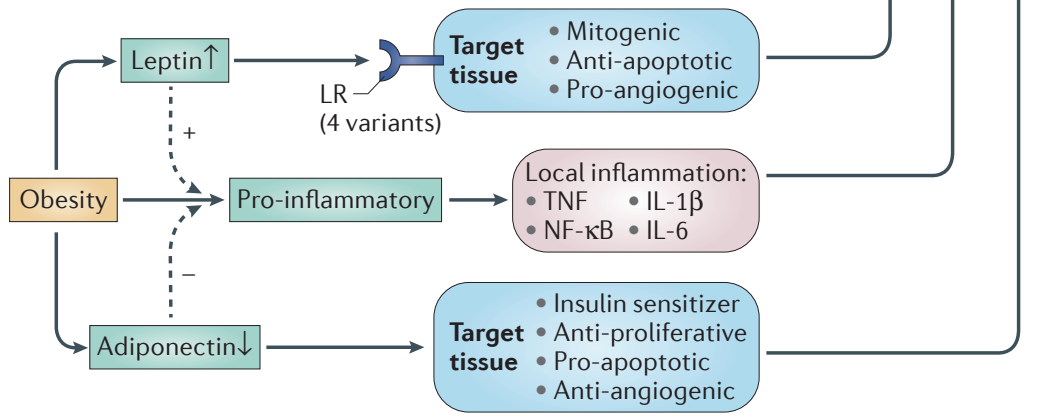

Figure 1 | Biological mechanism hypotheses. Schematic representation of the three main mechanisms that are hypothesized to link excess adiposity and cancer risk. Dashed arrows indicate indirect actions. $\Delta 4 \mathrm{~A}, \Delta 4$-androstenedione; $17 \beta-\mathrm{HSD}$, $17 \beta$-hydroxysteroid dehydrogenase; E1, oestrone; E2, oestradiol; IGF1, insulin-like growth factor I; IGF1R, IGF1 receptor; IGFBP, IGF-binding protein; IL, interleukin; $\mathrm{IR}$, insulin receptor; LR, leptin receptor; NF- $\mathrm{B}$, nuclear factor- $\mathrm{kB}$; SHBG, sex hormone-binding globulin; T, testosterone; TNF, tumour necrosis factor. analysis, the association of BMI with postmenopausal breast cancer risk was almost entirely explained by the increase in oestradiol levels with higher $\mathrm{BMI}^{66}$.

Adiposity is negatively correlated with testosterone concentrations in men $^{68}$ but positively correlated in women ${ }^{66}$. The EHCCBG ${ }^{65}$ and EPIC ${ }^{67}$ analyses demonstrated that elevated blood concentrations of androgens are associated with increased risk of breast cancer in both premenopausal and postmenopausal women. Thus, androgens may be potential candidates linking obesity and breast cancer. However, the experimental evidence is conflicting: the conventional wisdom is that androgens inhibit normal breast growth but, in animal and cultured cell experiments, androgens may have either inhibitory or stimulatory effects (reviewed elsewhere ${ }^{64}$ ).

For endometrial cancer, increased oestradiol levels not only increase endometrial cell proliferation and inhibit apoptosis (attributes that favour tumorigenesis) but also stimulate the local synthesis of IGF1 in endometrial tissue ${ }^{5}$. Indeed, the proliferative actions of oestradiol on endometrial tissue are mainly mediated by an increase in the local production (mostly in uterine tissue) of IGF1. Progesterone diminishes oestrogenic action in the endometrium by stimulating oestradiol metabolism and inducing the synthesis of IGF-binding protein 1 (IGFBP1), which inhibits IGF1 (REF. 69). Endometrial cancer offers an example in which more than one biological mechanism might link obesity with increased cancer risk. Similarly to studies for breast cancer risk, epidemiological studies support an unopposed oestrogen hypothesis for endometrial cancer, but ovarian hyperandrogenism (and concomitantly reduced progesterone levels) may also play a part ${ }^{69}$.

Androgens are important in the maturation of the prostate gland, but prospective studies assessing the associations between circulating sex hormone concentrations and prostate cancer risk have reported inconsistent findings. In men, compared with normal weight, obesity is associated with lower mean concentrations of serum testosterone, so how can sex hormones explain the observed links between obesity and advanced-stage prostate cancer? It is speculated that a low-testosterone environment (paradoxically) favours the development of a less-differentiated, aggressive cancer phenotype ${ }^{70}$, although the supporting evidence is currently limited and indirect. The Prostate Cancer Prevention Trial ${ }^{71}$ reported that finasteride, which lowers dihydrotestosterone levels, decreased risk of well-differentiated prostate cancer while simultaneously being associated with an increased risk of high-grade prostate cancer (Gleason grade $\geq 7$ ). Two prospective studies ${ }^{72,73}$ reported that low concentrations of testosterone in serum were associated with a higher risk of poorly differentiated prostate cancer; these findings need corroboration and adjustment for BMI.

There are limitations to the sex hormone hypothesis. First, circulating sex hormone levels change cyclically in premenopausal women, making it challenging to measure a 'steady state' of risk or risk modification. Second, the focus is on endocrine effects, but it is increasingly appreciated that paracrine mechanisms are key to 
the link between adiposity and cancer risk (discussed below), particularly for breast cancer ${ }^{61,74-76}$. Third, there are multiple potential candidate sex hormones: it is unclear which of these are key drivers and which are bystanders. Fourth, the hypothesis predicts the strongest associations with hormone-receptor-positive $\left(\mathrm{ER}^{+} \mathrm{PR}^{+}\right)$ breast cancer; however, data show that an elevated BMI is equally associated with $\mathrm{ER}^{-} \mathrm{PR}^{-}$tumours among postmenopausal women who have never used $\mathrm{HRT}^{42}$. Fifth, the hypothesis is relevant for sex-hormone-sensitive tumours in women, but it is less clear whether it explains links between adiposity and advanced prostate cancer in men. Furthermore, it is unclear whether this hypothesis is applicable to tumours conventionally not included in the list of sex-hormone-sensitive cancers. For example, BMI-cancer associations are weaker in women than in men for colorectal cancer, and exogenously administered oestrogen-rich HRT is associated with reduced risk of colorectal cancer ${ }^{77}$, but whether endogenous physiological-range oestrogen-related hormones offer some degree of attenuation of the BMI-cancer association in women is speculative.

Insulin resistance and IGFs. Circulating insulin levels positively correlate with increasing BMI, and many obese individuals are insulin resistant. Two decades ago, McKeown-Eyssen ${ }^{78}$ and Giovannucci ${ }^{79}$ suggested that hyperinsulinaemia may contribute to cancer development through two pathways: direct growth-promoting signalling of elevated levels of insulin, and indirectly through what has come to be known as the insulin-IGF hypothesis. The latter postulates that prolonged hyperinsulinaemia reduces production of IGFBP1 and IGFBP2 (which normally bind IGF1 and inhibit its action), with resultant increases in the levels of free or bioactive IGF1 and concomitant changes in the cellular environment that favour tumour development. Activation of the insulin receptor (IR) and IGF1 receptor (IGF1R) triggers cancer-relevant intracellular signalling cascades including those required for mitogenesis, anti-apoptosis, angiogenesis and tumour-associated lymphangiogenesis, which favour tumour development and spread ${ }^{80}$.

In parallel, a large volume of epidemiological studies have examined the relationships between circulating total IGF1 and cancer risk. Our meta-analyses ${ }^{81,82}$ of these studies support relationships between total IGF1 levels and the risk of developing prostate, colorectal, premenopausal breast and postmenopausal breast cancer. Initial studies reported inverse associations between circulating levels of IGFBP3 (the signalling of which is thought to have anti-apoptotic effects) and cancer risk, but subsequent epidemiological studies did not replicate these findings ${ }^{82}$.

There are several shortfalls to the insulin-IGF hypothesis. Considering the insulin component: first, in epidemiological studies, the measurement of serum insulin levels is highly dependent on the state and duration of fasting, assay characteristics and genetic factors, and, accordingly, surrogates of insulin secretion (for example, C-peptide) or of insulin resistance (for example, homeostasis model assessment) are often used. It is now recognized that these surrogates are poor indicators of individual insulin resistance ${ }^{83}$. Determination of plasma glucose levels 2 hours after an oral glucose load is an alternative surrogate of insulin resistance, but currently the resource-heavy assessment of this exposure (as a continuous measure) with cancer has been limited to the end point of cancer-associated mortality in the DECODE collaboration ${ }^{84}$. Second, although there is a large volume of animal model data supporting the link between supraphysiological levels of insulin and tumour promotion ${ }^{85}$, these insulin levels are not applicable in humans and the direct tumour-development effect attributable to insulin is probably very modest. Third, the biology of insulin and IGF1R systems is complex. Hybrid receptors of IR and IGF1R exist that bind insulin and IGF1, and IR may exist as isoform A (IR-A) and isoform B (IR-B). In many common adult malignancies, IR expression tends to be almost exclusively IR-A. IGF1 has stronger binding affinity for IR-A compared with IR-B and, on theoretical grounds, much of the direct tumour-promoting action of IR may be through IGF1, rather than through insulin binding. Finally, if insulin signalling is invoked as a candidate mechanism to explain adiposity and cancer risk, one would expect that exogenously administered insulin in patients with diabetes might be associated with increased cancer risk. Although epidemiological studies raised this concern in the late 2000s, it is now appreciated that these associations spuriously resulted from methodological limitations in the study analyses ${ }^{86}$, and they failed to emerge in a secondary analysis of the large ORIGIN trial of the long-acting insulin analogue glargine versus standard of care ${ }^{87}$.

Considering the IGF1 component: first, in mice, total levels of IGF1 increase with increasing fatness across the range of weights; however, this is not true for humans, in whom total levels of IGF1 increase only to a BMI of approximately $27 \mathrm{~kg}$ per $\mathrm{m}^{2}$, thereafter declining with increasing weight ${ }^{88}$. Second, in the human circulation, IGF1 is heavily bound to various IGFBPs. The structural relationship of IGF1 to IGFBPs differs in tissue, and there is no direct evidence that circulating IGF1 levels correlate with cellular downstream pathways at a tissue level. Third, a review of epidemiological studies has raised concerns that there may be reporting bias for studies describing significant associations between either insulin or IGF1 and cancer risk ${ }^{89}$. Finally, in overweight individuals who intentionally lose weight, total and free IGF1 concentrations tend to increase (discussed below) ${ }^{90}$.

Adipokine pathophysiology and systemic inflammation. Polypeptide hormones derived from adipocytes are known as adipokines ${ }^{91}$. There are more than 50 fifty different types of adipokines; leptin and adiponectin are the types most studied in the context of cancer risk. Adipokines are associated with the inflammatory system; for example, leptin is a potent inflammatory agent, whereas adiponectin has potent anti-inflammatory activity.

Systemic leptin concentrations are proportional to the amount of body fat, as insulin induces leptin gene expression to signal suppression of appetite. Mean levels 
Visceral adipose tissue (VAT). Adipose tissue arising in the abdominal cavity namely, the omentum, mesenteric and retro-peritoneal fat stores - but excluding within-viscera fat, such as intrahepatic and intrapancreatic fat.

Biomarkers

Characteristics that are objectively measured and evaluated as indicators of normal biological processes, pathogenic processes or pharmacological responses to a therapeutic intervention. of insulin are higher in women than in men $^{92}$. Leptin is potentially relevant for cancer development: it has pleiotropic effects, it is mitogenic (notably, in breast, colon and prostate cancer cell lines), it is anti-apoptotic, it mediates immune suppression, and it is pro-angiogenic by itself and in synergy with vascular endothelial growth factor (VEGF) ${ }^{93,94}$. The leptin receptor occurs as at least four splice variants, but only the long form (LRb) has an intracellular domain of sufficient length to provide full signal-transducing capabilities ${ }^{95}$. LRb activates PI3K, MAPK and signal transducer and activator of transcription (STAT) signalling, which are critical pathways involved in cell survival, proliferation and differentiation $^{62}$. Despite this repertoire of mechanisms, epidemiological studies assessing associations between serum leptin concentrations and cancer risk have reported inconsistent findings (reviewed elsewhere ${ }^{96,97}$ ).

Adiponectin is the most abundant adipokine, secreted mainly from visceral adipose tissue (VAT). Unlike leptin, it is produced only by mature adipocytes. Serum adiponectin levels are negatively correlated with BMI and, in turn, insulin and oestrogens may suppress adiponectin secretion ${ }^{98}$. After adjustment for BMI, serum concentrations of adiponectin are higher in women than in men, and higher in postmenopausal than in premenopausal women ${ }^{99}$. Of importance to tumour development, adiponectin may have indirect effects, sensitizing cells to insulin or through anti-inflammatory actions. It may also have direct effects: adiponectin sequesters growth factors at the pre-receptor level or by binding to adiponectin receptor 1 (ADIPOR1) and ADIPOR2, activating AMP-activated protein kinase (AMPK) while inhibiting ERK1 and ERK2, PI3K-AKT, WNT- $\beta$-catenin, nuclear factor- $\kappa \mathrm{B}(\mathrm{NF}-\kappa \mathrm{B})$, and Janus kinase 2 (JAK2)-STAT3 signalling. Collectively, these effects result in reduced fatty acid and protein synthesis, decreased cellular growth, proliferation and DNA mutagenesis and increased cell cycle arrest and apoptosis, thus negatively influencing carcinogenesis (reviewed elsewhere ${ }^{100}$ ). Against this biological background, a comprehensive review ${ }^{100}$ of the epidemiological data shows broadly consistent inverse associations between circulating adiponectin concentrations and risk of obesityassociated cancers, including endometrial, breast, advanced prostate, colorectal, renal, and pancreatic (mainly in never smokers) cancers but not lung cancer (see Supplementary information S1 (table)).

Adiposity is associated with a state of chronic (subclinical) inflammation. As adipose tissue expands, the levels of $\mathrm{C}$-reactive protein (CRP), tumour necrosis factor (TNF), interleukin-1 $\beta$ (IL-1 $\beta$ ), IL-6 and IL-18 increase ${ }^{101-103}$. An early epidemiological study showed an association of CRP levels with colorectal cancer ${ }^{104}$, but a subsequent meta-analysis of eight prospective studies reported only modest correlations ${ }^{105}$. Later studies showed either positive associations ${ }^{106,107}$ or no relationship ${ }^{108}$.

Here again, there are several caveats and shortfalls. First, the molecular assembly of adiponectin is complex. There are three major oligomeric forms of adiponectin: a low-molecular-weight (LMW) trimer, a middle-molecular-weight (MMW) hexamer and a high-molecular-weight (HMW) multimer. LMW oligomers are the predominant form in the circulation, whereas the majority of intracellular adiponectin consists of HMW multimers. The ratio of HMW to LMW is critical to insulin sensitivity. The biological effects of adiponectin depend on not only relative circulating concentrations but also its form and the tissue-specific expression of its receptor subtypes (ADIPOR1 and ADIPOR2). Both adiponectin receptors may exist in tumour cells ${ }^{109}$, but more studies are needed to characterize these subtypes and their functions. Second, studies in fatless A-Zip/F1 mice, which have undetectable adipokine levels in the circulation but display accelerated tumour formation, suggest that adipose tissue is not essential for tumour development ${ }^{110}$. Third, a review of epidemiological studies of biomarkers and subsequent cancer risk has raised concerns about biased reporting in studies of inflammation biomark$\mathrm{ers}^{89}$. Circulating levels of adipokines and inflammatory markers fluctuate considerably in the presence of chronic diseases, differing diets, medications and differing levels of physical activity ${ }^{62,111}$.

Against these background inconsistencies, there is a continuing need to refine existing hypothesized mechanisms for the link between adiposity and cancer as well as to find additional mechanisms that explain the specificity of the epidemiological observations.

\section{Weight loss and cancer-relevant biomarkers}

Weight-losing interventions may affect intermediary biomarkers. Thus, observations from weight-losing studies could implicate some of the above hypotheses in obesity-associated cancer risk. Byers and Sedjo ${ }^{112}$ published a comprehensive review of studies evaluating changes in cancer-relevant risk biomarkers, namely: oestrogens, SHBG, IGF1, IGFBPs and the inflammatory markers CRP, IL-6 and TNF. Their findings were as follows. First, oestrogen levels drop and SHBG levels increase coincident with intentional weight loss, with about a one-third reduction in free oestradiol to be expected from a $10 \%$ weight loss. Second, CRP levels drop by about one-third after weight loss. Third, reductions in TNF and IL-6 levels are consistently seen but are of a smaller magnitude. Fourth, changes in levels of IGF1 and IGFBPs are small and may be in either direction.

It is possible that the observed inconsistencies and small effects reflect measurement errors (in the assays) or chance findings in small sample sizes. An alternative and more plausible explanation is that the lack of substantial change in some intermediary biomarkers (for example, IGF1) in a cancer-protective direction, in the face of substantial weight reduction, argues against that biomarker being a key intermediary in obesityassociated carcinogenesis. One recent large randomized trial of dietary and exercise interventions reported no significant changes in IGF1 and IGFBP3, despite substantial reductions in body weight in the experimental groups ${ }^{113}$, leading the authors to remark that: "modified IGF-1 bioavailability is unlikely to be a mechanism through which caloric restriction reduces cancer risk". 


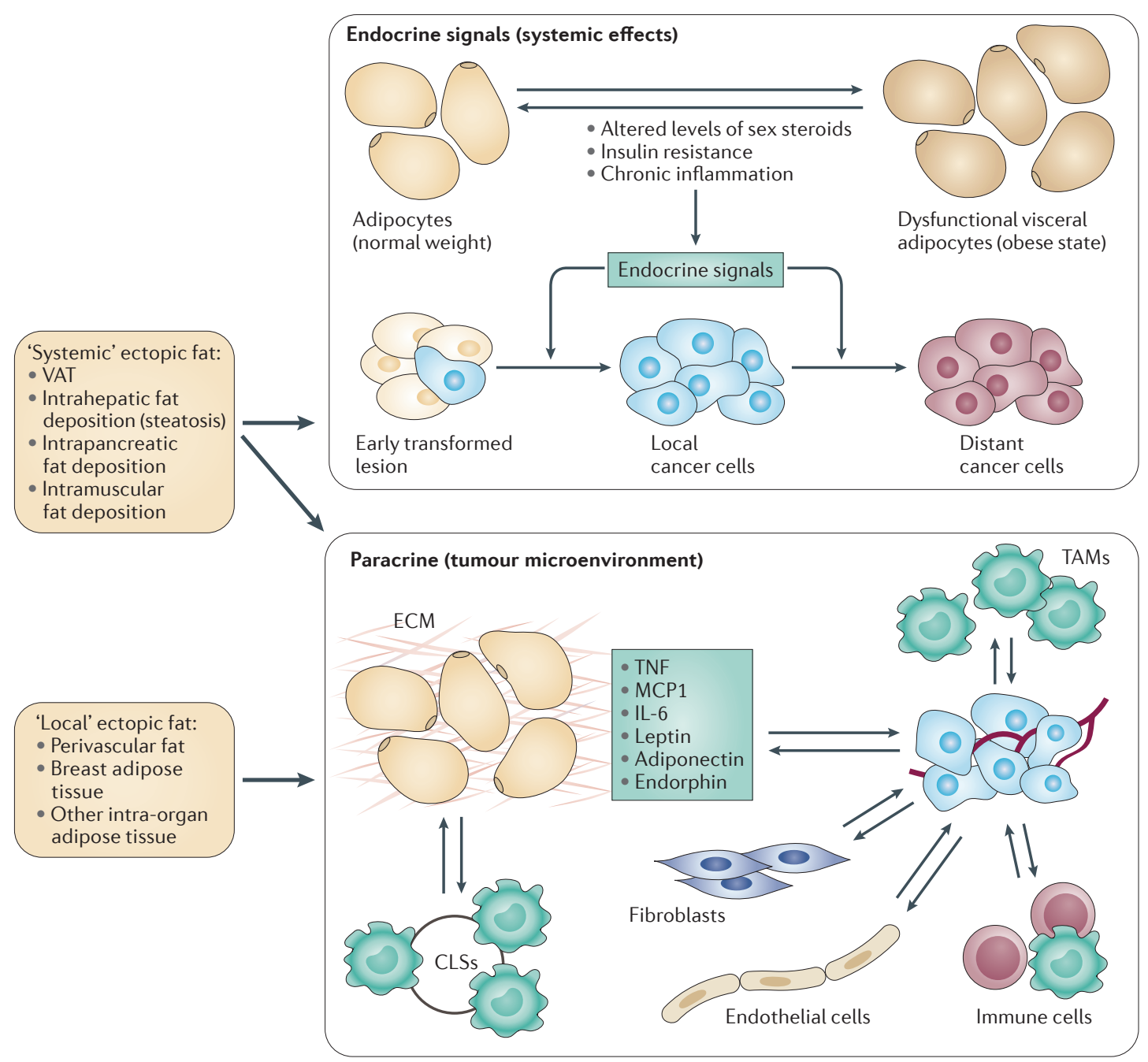

Figure 2 | The roles of ectopic fat. Schematic representation of ectopic fat depots with systemic and local effects. The expansion of local adipocytes fuels infiltration of adipose tissue by macrophages, the phenotype of which shifts towards the inflammatory M1 type ${ }^{103}$. Monocyte chemoattractant protein 1 (MCP1; also known as CCL2) is a key molecule that mediates this macrophage infiltration ${ }^{138}$. Additionally, the number of regulatory $T$ cells decreases while the number of $C D 8^{+} T$ cells increases, promoting further macrophage recruitment. CLSs, crown-like structures; ECM, extracellular matrix; IL-6, interleukin-6; TAMs, tumour-associated macrophages; TNF, tumour necrosis factor; VAT, visceral adipose tissue.

\section{Body fat distributions and cancer risk}

BMI does not fully capture the complex biology of adiposity. Excess body fat is a heterogeneous condition in which individuals with similar BMIs may have distinct metabolic and cardiovascular disease risk ${ }^{114}$. Variation in body fat distribution provides a potential explanation for some of the risk differential that persists after accounting for BMI and standard disease risk factors ${ }^{115}$. Increasingly, it is recognized that a proportion of overweight or obese individuals might not be at an increased risk for metabolic complications of obesity and have a phenotype referred to as metabolically healthy obesity (MHO), which contrasts with the metabolically unhealthy obesity (MUO) phenotype ${ }^{116}$. This new classification is driving treatment algorithms in individuals at risk of cardiovascular disease and type 2 diabetes and may have an application in cancer epidemiology. For example, results from the
Framingham Heart Study ${ }^{117}$ suggest that cancer risk may be lower among $\mathrm{MHO}$ older adults than among MUO individuals.

Classification of types of ectopic fat. Body fat distribution differs between MHO and MUO individuals: for example, $\mathrm{MHO}$ individuals have less local ectopic fat than MUO individuals. The clinical importance of local ectopic adipose tissue depots, which surround organs and blood vessels or are located within organs, is now well recognized, as these depots are key risk factors for cardiovascular disease ${ }^{118}$. Thus, understanding and classifying ectopic fat depots (systemic and local) is important (FIG. 2).

Sites with predominantly systemic effects include VAT and intramuscular fat: these have a well-established role in the development of cardiovascular disease ${ }^{119}$. With the development of obesity, VAT is infiltrated 
Central adiposity

The storage of adipose tissue preferentially in adipocytes within the trunk rather than the extremities

\section{Steatosis}

The accumulation of intracellular fats, mainly triglycerides. Used typically to describe fat deposition within the liver (hepatic steatosis) but may equally apply to fat accumulation in other organs. by macrophages, and there is dysregulation of various fatsecreting factors that are important in the development of a subclinical systemic inflammation state and insulin resistance ${ }^{120}$. Although volumes of intrahepatic and intramuscular fats are much smaller than those of VAT, they may exert 'overspill' effects and contribute to systemic metabolic disease, and they are key determinants of insulin resistance ${ }^{121}$.

In contrast to ectopic fat with predominantly systemic effects, local fat depots surrounding the heart, blood vessels and kidneys correlate more strongly with metabolic disease states in the respective organs ${ }^{118}$. For example, pericardial fat has a stronger correlation than VAT has with coronary artery disease. This paradigm is now extending to cancer biology. For example, breast adipose tissue is an established ectopic fat depot with potential local effects on cancer development ${ }^{61}$.

Systemic ectopic fat (central adiposity) and cancer risk. WC was one of the earliest means of quantifying body fat distribution, as an approximation of central adiposity. With ageing, individuals lose lean body mass and gain weight as VAT. Metabolically active visceral fat releases substantial amounts of growth factors, inflammatory markers, free fatty acids (contributing to insulin resistance) $)^{122}$, locally produced oestrogen and adipokines, which might contribute to the development of diseases, including cancer. In epidemiological studies, individuals with larger amounts of visceral fat, as identified by their larger WC, have a higher risk of cardiovascular disease and type 2 diabetes than individuals with smaller amounts of VAT. In turn, anthropometric measures of central adiposity (such as WC and WHR) correlate more strongly than BMI with visceral fat, and they are thought by some investigators to be better indicators of cancer risk ${ }^{123}$.

Early studies supported the above hypothesis. For example, for colorectal cancer, in prospective studies in which both BMI and WC (or WHR) were measured and risk estimates determined for the development of colorectal cancer, risk seemed to be greater for WC in both genders ${ }^{124}$, or in women ${ }^{125}$. Furthermore, adjustment for BMI did not seem to attenuate associations ${ }^{124,125}$. Similarly, for postmenopausal breast cancer, early results from the Iowa Women Health Study ${ }^{126}$ suggested a statistically significant multiplicative interaction between age, BMI and WHR. However, in subsequent reports that specifically tested interactions between WC and BMI, in relation to colorectal ${ }^{127,128}$ and breast ${ }^{40,129,130}$ cancer risk, statistically significant associations were not found. For more recently reported prospective studies evaluating the risk of developing other cancers in which both BMI and WC (or WHR) were measured and risk estimates determined, summary risk estimates from WCRF/AICR pooled analyses were broadly similar for equivalent increments of BMI and WC in cancers of the pancreas $^{131}$, endometrium ${ }^{132}$ and ovaries ${ }^{9}$, and advanced prostate cancer ${ }^{17}$.

Taken together, there is limited evidence that WC (or WHR) is a better predictor of cancer risk than BMI. Thus, as visceral adiposity is a key determinant of insulin resistance, by extension, one would hypothesize that surrogates of central adiposity - for example, WC and WHR - are stronger predictors than BMI for cancer risk. However, the epidemiological evidence does not support this hypothesis. The current explanation is that WC is a poor approximation of central adiposity, as it captures both subcutaneous adipose tissue (SAT; which is typically non-ectopic) and VAT (which is typically ectopic) (see Supplementary information S2 (table)), and there is a need to develop techniques to better quantify these separate fat depots ${ }^{18}$.

Local ectopic fat and cancer development. The theory of excess adipose tissue having a local toxic effect is supported by multiple lines of evidence from translational research ${ }^{133}$ and epidemiology ${ }^{134}$. This theory is now increasingly relevant for the development of breast cancer and probably other types, such as hepatocellular carcinoma and pancreatic cancer ${ }^{18}$. The amount of fat at these sites is proportional to the total body fat mass ${ }^{18,135}$, but these fat depots retain residual independence and thus might be more relevant than total body adiposity to local tumour development. In breast cancer, the cellular and metabolic makeup of the local adipose tissue is additionally important for tumour progression and metastasis (reviewed elsewhere ${ }^{61,136,137}$ ).

A hallmark of local ectopic fat deposition is local inflammation ${ }^{103,138}$ (FIG. 2). The inflammatory mediators secreted by macrophages not only act locally, in a paracrine manner, but also may contribute to general systemic inflammation and promote an environment that favours tumour development. One clear example of this 'mirroring effect' is seen in the liver (and the hypothesized pathway to obesity-associated hepatocellular carcinoma). Hepatic steatosis is the intrahepatic accumulation of fats, commonly as a consequence of obesity, and may be a driver of insulin resistance and systemic inflammation ${ }^{121,139}$. Nutritional insults induce reactive oxygen species, leading to the production of pro-inflammatory cytokines and the recruitment of immune cells to the liver, and eventually to nonalcoholic fatty liver disease (NAFLD) ${ }^{140}$. NAFLD may progress to nonalcoholic steatohepatitis (NASH), a major risk factor for hepatocellular carcinoma, thus providing a possible link between obesity, insulin resistance, inflammation and the development of hepatocellular carcinoma (FIG. 3). There are several animal models that support this paradigm ${ }^{141,142}$. However, this process is not well understood, and the epidemiology is difficult to study ${ }^{143}$ owing to the lack of a reliable, noninvasive tool to quantify NAFLD and to the presence of strong confounders (which have often not been measured in the epidemiological studies of this to date), such as viral infection and alcohol consumption ${ }^{144}$.

Intrapancreatic fat, termed pancreatic steatosis, is an additional example of intra-organ fat deposition that increases with BMI ${ }^{145}$. Inflammation is a putative mechanism in the development of pancreatic cancer and a candidate obesity-driven pathway. In mice, excess fat intake leads to inflammation within the pancreas, leading to progression from normal pancreatic epithelium to pancreatic intraepithelial neoplasia, a precursor lesion 
White adipose tissue (WAT). A type of body fat. Mammals have three types of body fat: white, brown and beige. The main function of WAT is metabolism. of pancreatic cancer ${ }^{146}$. Here, again, an obesity-induced local inflammatory microenvironment seems to be important. Human data are just beginning to emerge to support this hypothesis ${ }^{147}$.

\section{New mechanistic hypotheses}

Intriguing new biological mechanisms are beginning to emerge that may be additional links between obesity and cancer risk.

Migrating adipose progenitor cells. The tumour stroma includes many different mesenchymal stromal cells (MSCs), which are important as progenitor cells for the formation of, for example, endothelial cells that are required for neovascularization. Intriguingly, MSCs are present in the circulation at low levels and may be recruited to tumour sites by pathological signals, such as hypoxia or inflammation (at least in mice) ${ }^{148}$. When recruited to the tumour, they become tumour stromal cells (also known as cancer-associated fibroblasts (CAFs)) and promote angiogenesis and drive tumour progression. Bone marrow was thought to be the main source of these circulating progenitor cells, but evidence indicates that they may arise from other sources, including white adipose tissue (WAT) ${ }^{148}$. In turn, WAT is expanded in obesity, which may then be a link between the systemic fat volumes and local ectopic fat mechanisms.

The microbiome, obesity and cancer. Intestinal microbiota exist in a symbiotic relationship with their host, by metabolizing compounds that the host is unable to utilize and controlling the balance of the immune responses in the host. However, the composition of the intestinal microbiome varies with diet and pathophysiological states, including obesity ${ }^{149}$. Recently developed -omics technologies to capture microbial data provide new insights into the roles of intestinal microorganisms and their metabolism and, for example, suggest that the intestinal microbiota contribute to colorectal carcinogenesis (at least in mice) ${ }^{150}$, via the influence of their metabolites. Importantly, it is now appreciated that gut microbial metabolites 'spill over' into the host's circulation

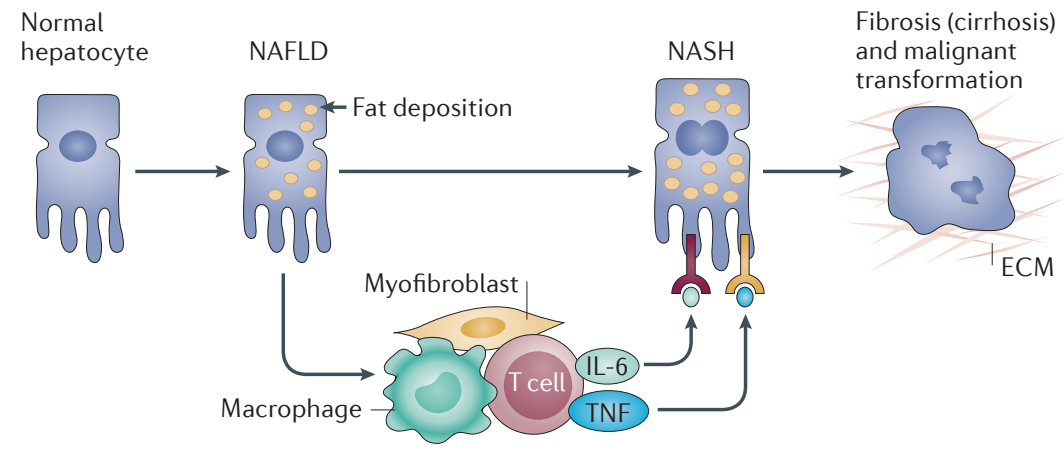

Figure 3 | Hypothesized steatosis-hepatocellular carcinoma pathway. In the absence of common hepatic insults, such as excess alcohol, accumulation of fat in the liver (nonalcoholic fatty liver disease (NAFLD)) is associated with chronic inflammation, known as nonalcoholic steatohepatitis (NASH). In some subpopulations, over time, NASD may progress either directly to hepatocellular carcinoma or indirectly through a cirrhosis state. ECM, extracellular matrix; IL-6, interleukin-6; TNF, tumour necrosis factor. and are involved in the pathogenesis of cancers distant from the gastrointestinal tract ${ }^{151}$. This field is still new and links with cancer remain speculative but will require consideration in the future.

\section{Future directions}

More-detailed measures of body fat deposition. If local ectopic fat deposition ${ }^{61}$ and organ-specific fat deposition are indeed relevant for cancer development, the challenge is now to accurately quantify these using noninvasive modalities in large-epidemiological-scale studies, including, for example, magnetic resonance imaging and ${ }^{1} \mathrm{H}$ magnetic resonance spectroscopy (illustrative examples are provided in Supplementary information S2 (table)). In the Framingham Heart Study ${ }^{152}$, more than 3,000 individuals already underwent ectopic fat quantification using multi-detector computed tomography scanning, with correlations to cardiovascular disease end points ${ }^{152}$. Large-scale imaging is planned with the UK Biobank and the German Cohort study, with opportunities to correlate with cancer outcomes ${ }^{18}$.

Causal relationships and Mendelian randomization. Up to this point, we have assumed that the associations between increased adiposity and cancer risk are causally related. Statistical associations between an exposure and an outcome in observational epidemiology can be spuriously produced by common causes of the exposure and the outcome, an effect known as confounding ${ }^{153}$. An example of this might be that increased adiposity is caused by other risk factors that cause cancer - such as increased energy intake, decreased physical activity or increased alcohol consumption - while obesity per se might have no causal effect on cancer risk (discussion expanded in Supplementary information S3 (table) using directed acyclic graphs (DAGs)). In practice, many epidemiological studies have adjusted for these confounders and shown that associations between adiposity and cancer risk remain, although residual unmeasured confounding may exist and adjustment for confounders might be only partial, as confounders might be measured with error.

Mendelian randomization may offer a solution to the problem of residual confounding, as it enables, under certain conditions, estimation of causal effects in observational studies using genetic variants as instrumental variables ${ }^{154}$. With a genetic variant taking the role of an instrumental variable (owing to the random assignment of alleles in gamete formation) one might, for example, estimate the average effect of the genetic variant on BMI difference and, in a second analysis, on disease risk. In turn, this would allow estimation of the increase in disease risk per unit increase in BMI. However, this would be possible only if the genetic variant (the instrumental variable) affects cancer risk only through its effect on $\mathrm{BMI}^{155,156}$ (BOX 1). More than 50 gene loci have been associated with the development of obesity through genome-wide association studies but few Mendelian randomization studies have been undertaken to date $e^{157,158}$. These focused on few gene loci and cancers, including polymorphisms in the CYP19A1 gene (which encodes aromatase) ${ }^{159}$, 


\section{Box 1 | Mendelian randomization, obesity and cancer risk}

Observational epidemiology suggests that obesity may be associated with increased risk of several cancer types, but such studies may have several problems, including confounding, reverse causation, selection bias and attenuation by measurement errors (regression dilution bias). Mendelian randomization may help to address these limitations.

Mendelian randomization studies use genetic variants as proxies of non-genetic risk factors to assess whether a risk factor is causally related to a disease. The approach exploits the independent randomized assortment of maternal and paternal alleles at the time of gamete formation to remove confounding and other biases. Instrumental variable analysis is used to obtain estimates for the association of the risk factor with the disease that can have a causal interpretation.

Genome-wide association studies (GWASs) reported in the past few years have revealed more than 50 gene loci associated with the development of obesity. Importantly, some loci predict a higher body mass index (BMI), and others are associated with central adiposity (as approximated by waist circumference (WC)).

Future analyses might make causal inferences through assessment of dispositional propensities (for example, tendency to get fatter), characterizing 'difficult to measure' exposures (for example, insulin resistance at a population level), and inter-generational influences.

\section{Bias}

A systematic deviation of a result from a true value.

Structural equation

modelling

A family of statistical modelling techniques that combine latent variables through regression equations.
CRP single nucleotide polymorphisms (SNPs) ${ }^{160}$ and obesity-associated genes and SNPs - fat mass and obesityassociated (FTO), rs9939609, melanocortin 4 receptor $(M C 4 R)$ and rs17782313 in endometrial cancer ${ }^{161}$, FTO and rs9939609 in prostate cancer ${ }^{162}, M C 4 R$ in colorectal cancer ${ }^{163}$, and 28 obesity-related SNPs in colorectal cancer $^{164}$ - but no consistent pattern has emerged.

Other modelling approaches. Several alternative modelling approaches might be considered in this field to increase the power of causal inference for obesitycancer associations. Examples include structural equation modelling (which explores the extent to which the effect of an exposure on a disease risk acts through a putative mediator or mechanism: that is, it decomposes a total effect into an indirect (mediated) effect and a direct effect ${ }^{165}$ ) using repeated measurements of, for example, BMI, and life-course analyses with $\mathrm{BMI}$ as a time-varying variable ${ }^{117}$. A further example is a holistic or factor analysis approach, as used in the EPIC cohort to clarify the role of obesity-associated biomarkers, with endometrial cancer as the cancer of interest ${ }^{166}$. This analysis 'clustered' related intermediary biomarkers into families and, for example, showed that members of the lipids were not associated with endometrial cancer risk.

\section{In summary}

We have identified four broad concepts. First, the epidemiological evidence shows specificity of associations between measures of adiposity and cancer risk. This indicates that there is no one mechanism (or set of mechanisms) that fits all. Second, the specificity of associations (demonstrated by the epidemiology) argues for a paradigm shift towards local tissue-specific mechanistic rather than global systemic physiological explanations. Third, the weight-reduction clinical trial data indicate that there is a range of sizes and directions of change in intermediary biomarkers, which in turn inform the mechanistic importance (or lack of importance) of a given proposed pathway. Fourth, the current epidemiological data fail to demonstrate striking differences in cancer risk associations for BMI (as an approximation of total body adiposity) versus other anthropometric measures (as approximations of specific body compartments). The most likely explanation is that current measures of body fat distribution (for example, WC) are too crude, and there is a continuing need for better characterization. At present, the most optimistic quantification modalities will come through large-scale imaging projects. These epidemiological approaches will help to identify dominant mechanistic pathways and develop future cancer-specific targeted preventive strategies.

\section{Note added in proof}

Since this Review was accepted for publication, a largescale study has been published demonstrating the utility of Mendelian randomization in the evaluation of the mechanisms linking obesity and cancer risk ${ }^{167}$, which relates to the discussion in BOX 2. From a consortium of studies from European ancestry populations, and using used SNPs associated with fasting insulin (18 variants), early insulin secretion (17 variants) and BMI (32 variants) as instrumental variables, this analysis (of 1287 cases and 8273 controls) provides evidence to support a causal association of higher insulin levels, independent of BMI, with endometrial cancer risk (cases, 1287: controls, 8273).
1. Renehan, A., Tyson, M., Egger, M., Heller, R. F. \& Zwahlen, M. Body mass index and incidence of cancer: a systematic review and meta-analysis of prospective observational studies. Lancet 371, 569-578 (2008). A dose-response meta-analysis standardized across 20 cancer types, demonstrating sex- and site-specific associations between BMI and cancer risk.

2. World Cancer Research Fund and American Institute for Cancer Research. Food, Nutrition, Physical Activity, and the Prevention of Cancer: a Global Perspective (AICR, 2007).

A series of systematic reviews establishing that body fatness is a risk factor for incidence of several cancer types.

3. Renehan, A. G. Bariatric surgery, weight reduction and cancer prevention. Lancet Oncol. 10, 640-641 (2009).

4. Arnold, M. et al. Global burden of cancer attributable to high body-mass index in 2012: a population-based study. Lancet Oncol. 16, 36-46 (2014).

An analysis of the global burden of cancer attributable to high BMI. Reports that $3.6 \%$ of all new cancers in adults are attributable to high BMI.
5. Calle, E. E. \& Kaaks, R. Overweight, obesity and cancer: epidemiological evidence and proposed mechanisms. Nat. Rev. Cancer 4, 579-591 (2004). A seminal review on obesity and cancer risk, setting down the main biological hypotheses underpinning the link.

6. Sperrin, M., Marshall, A. D., Higgins, V., Buchan, I. E. $\&$ Renehan, A. G. Slowing down of adult body mass index trend increases in England: a latent class analysis of cross-sectional surveys (1992-2010). Int. J. Obes. 38, 818-824 (2014).

7. Aune, D. et al. Body mass index, abdominal fatness and pancreatic cancer risk: a systematic review and non-linear dose-response meta-analysis of prospective studies. Ann. Oncol. 23, 843-852 (2012).

8. Collaborative Group on Epidemiological Studies of Ovarian Cancer. Ovarian cancer and body size: individual participant meta-analysis including 25,157 women with ovarian cancer from 47 epidemiological studies. PLoS Med. 9, e1001200 (2012).

9. World Cancer Research Fund and American Institute for Cancer Research. Continuous Update
Project Report. Food, Nutrition, Physical Activity, and the Prevention of Ovarian Cancer (AICR, 2014).

10. Larsson, S. C. $\&$ Wolk, A. Body mass index and risk of non-Hodgkin's and Hodgkin's lymphoma: a metaanalysis of prospective studies. Eur. J. Cancer 47 2422-2430 (2011)

11. Koebnick, C. et al. Body mass index, physical activity, and bladder cancer in a large prospective study. Cancer Epidemiol. Biomarkers Prev. 17, 1214-1221 (2008).

12. Moore, S. C. et al. Height, body mass index, and physical activity in relation to glioma risk. Cancer Res. 69, 8349-8355 (2009)

13. Lerro, C. C., McGlynn, K. A. \& Cook, M. B. A systematic review and meta-analysis of the relationship between body size and testicular cancer. Br. J. Cancer 103. 1467-1474 (2010)

14. Bhaskaran, K. et al. Body-mass index and risk of 22 specific cancers: a population-based cohort study of 5.24 million UK adults. Lancet 384, 755-765 (2014). 
15. Freedland, S. J., Giovannucci, E. \& Platz, E. A. Are findings from studies of obesity and prostate cancer really in conflict? Cancer Causes Control 17, 5-9 (2006).

16. Discacciati, A., Orsini, N. \& Wolk, A. Body mass index and incidence of localized and advanced prostate cancer - a dose-response meta-analysis of prospective studies. Ann. Oncol. 23, 1665-1671 (2012).

17. World Cancer Research Fund and American Institute for Cancer Research. Continuous Update Project Report: Diet, Nutrition, Physical Activity, and Prostate Cancer (AICR, 2014).

18. Coe, P. O., O'Reilly, D. A. \& Renehan, A. G. Excess adiposity and gastrointestinal cancer. Br. J. Surg. 101, 1518-1531 (2014).

19. Keum, N. et al. Adult weight gain and adiposityrelated cancers: a dose-response meta-analysis of prospective observational studies. J. Natl Cancer Inst 107, dju428 (2015).

20. Smith, L. et al. Body mass index and risk of lung cancer among never, former, and current smokers. J. Natl Cancer Inst. 104, 778-789 (2012).

21. Steffen, A. et al. Anthropometry and esophageal cancer risk in the European prospective investigation into cancer and nutrition. Cancer Epidemiol. Biomarkers Prev. 18, 2079-2089 (2009).

22. Gaudet, M. M. et al. Prospective studies of body mass index with head and neck cancer incidence and mortality. Cancer Epidemiol. Biomarkers Prev. 21, 497-503 (2012)

23. Etemadi, A. et al. A prospective cohort study of body size and risk of head and neck cancers in the NIHAARP diet and health study. Cancer Epidemiol. Biomarkers Prev. 23, 2422-2429 (2014).

24. Akbartabartoori, M., Lean, M. E. \& Hankey, C. R. Relationships between cigarette smoking, body size and body shape. Int. J. Obes. 29, 236-243 (2005)

25. Renehan, A. G., Leitzmann, M. F. \& Zwahlen, M. Re body mass index and risk of lung cancer among never former, and current smokers. J. Natl Cancer Inst. 104 1680-1681 (2012).

26. Renehan, A. G. in Handbook of Obesity (eds Bray, G. $\&$ Bouchard, C.) 561-572 (Informa Healthcare, 2014).

27. Crosbie, E. J., Zwahlen, M., Kitchener, H. C., Egger, M. \& Renehan, A. G. Body mass index, hormone replacement therapy, and endometrial cancer risk: a meta-analysis. Cancer Epidemiol. Biomarkers Prev. 19, 3119-3130 (2010)

28. World Cancer Research Fund and American Institute for Cancer Research. Continuous Update Project Report. Food, Nutrition, Physical Activity, and the Prevention of Breast Cancer (AICR, 2010).

29. Eliassen, A. H. et al. Endogenous steroid hormone concentrations and risk of breast cancer among premenopausal women. J. Natl Cancer Inst. 98, 1406-1415 (2006).

30. Kaaks, R. et al. Serum sex steroids in premenopausa women and breast cancer risk within the European Prospective Investigation into Cancer and Nutrition (EPIC). J. Natl Cancer Inst. 97, 755-765 (2005).

31. Renehan, A. G. Obesity and cancer in Asia-Pacific populations. Lancet Oncol. 11, 704-705 (2010).

32. Huang, Z. et al. Dual effects of weight and weight gain on breast cancer risk. J. Am. Med. Associ. 278 1407-1411 (1997).

33. Kaaks, R. et al. Breast-cancer incidence in relation to height, weight and body-fat distribution in the Dutch 'DOM' cohort. Int. J. Cancer 76, 647-651 (1998).

34. Lahmann, P. H. et al. Body size and breast cancer risk findings from the European Prospective Investigation into Cancer and Nutrition (EPIC). Int. J. Cancer 111 762-771 (2004)

35. Sperrin, M., Marshall, A. D., Higgins, V., Renehan, A. G. \& Buchan, I. E. Body mass index relates weight to height differently in women and older adults: serial cross-sectional surveys in England (1992-2011). J. Public Health (Oxf.) fdv067 (2015)

36. Green, J. et al. Height and cancer incidence in the Million Women Study: prospective cohort, and metaanalysis of prospective studies of height and total cancer risk. Lancet Oncol. 12, 785-794 (2011).

37. Renehan, A. G. Height and cancer: consistent links, but mechanisms unclear. Lancet Oncol. 12, 716-717 (2011).

38. Suzuki, R., Orsini, N., Saji, S., Key, T. J. \& Wolk, A Body weight and incidence of breast cancer defined by estrogen and progesterone receptor status - a metaanalysis. Int. J. Cancer 124, 698-712 (2009).
39. Ahn, J. et al. Adiposity, adult weight change, and postmenopausal breast cancer risk. Arch. Intern. Med. 167, 2091-2102 (2007)

40. Canchola, A. J. et al. Body size and the risk of postmenopausal breast cancer subtypes in the California Teachers Study cohort. Cancer Causes Control 23, 473-485 (2012).

41. Harris, H. R., Willett, W. C., Terry, K. L. \& Michels, K. B. Body fat distribution and risk of premenopausal breast cancer in the Nurses' Health Study II. J. NatI Cancer Inst. 103, 273-278 (2011).

42. Ritte, R. et al. Adiposity, hormone replacement therapy use and breast cancer risk by age and hormone receptor status: a large prospective cohort study. Breast Cancer Res. 14, R76 (2012).

43. Setiawan, V. W. et al. Breast cancer risk factors defined by estrogen and progesterone receptor status: the multiethnic cohort study. Am. J. Epidemiol. 169 1251-1259 (2009)

44. Phipps, A. I. et al. Body size, physical activity, and risk of triple-negative and estrogen receptor-positive breast cancer. Cancer Epidemiol. Biomarkers Prev. 20, 454-463 (2011).

45. Yang, X. R. et al. Associations of breast cancer risk factors with tumor subtypes: a pooled analysis from the Breast Cancer Association Consortium studies. J. Natl Cancer Inst. 103, 250-263 (2011).

46. Feigelson, H. S. et al. Adult weight gain and histopathologic characteristics of breast cancer among postmenopausal women. Cancer 107, 12-21 (2006).

47. Palmer, J. R., Adams-Campbell, L. L., Boggs, D. A., Wise, L. A. \& Rosenberg, L. A prospective study of body size and breast cancer in black women. Cancer Epidemiol. Biomarkers Prev. 16, 1795-1802 (2007).

48. Potter, J. D. et al. Progesterone and estrogen receptors and mammary neoplasia in the lowa Women's Health Study: how many kinds of breast cancer are there? Cancer Epidemiol. Biomarkers Prev. 4, 319-326 (1995)

49. Suzuki, R., Rylander-Rudqvist, T., Ye, W., Saji, S. \& Wolk, A. Body weight and postmenopausal breas cancer risk defined by estrogen and progesterone receptor status among Swedish women: a prospective cohort study. Int. J. Cancer 119 1683-1689 (2006)

50. Allred, D. C., Brown, P. \& Medina, D. The origins of estrogen receptor $\alpha$-positive and estrogen receptor $\alpha$-negative human breast cancer. Breast Cancer Res. 6, 240-245 (2004)

51. Asselin-Labat, M. L. et al. Control of mammary stem cell function by steroid hormone signalling. Nature 465, 798-802 (2010)

52. Bokhman, J. V. Two pathogenetic types of endometrial carcinoma. Gynecol. Oncol. 15, 10-17 (1983)

53. Setiawan, V. W. et al. Type I and II endometrial cancers: have they different risk factors? J. Clin. Oncol. 31, 2607-2618 (2013).

54. Olsen, C. M. et al. Obesity and risk of ovarian cancer subtypes: evidence from the Ovarian Cancer Association Consortium. Endocr. Relat. Cancer 20 251-262 (2013)

55. Campbell, P. T. et al. Case-control study of overweight, obesity, and colorectal cancer risk, overall and by tumor microsatellite instability status. J. Natl Cancer Inst. 102, 391-400 (2010)

56. Satia, J. A. et al. Diet, lifestyle, and genomic instability in the North Carolina Colon Cancer Study. Cancer Epidemiol. Biomarkers Prev. 14, 429-436 (2005).

57. Slattery, M. L. et al. Associations between cigarette smoking, lifestyle factors, and microsatellite instability in colon tumors. J. Natl Cancer Inst. 92, 1831-1836 (2000)

58. Hughes, L. A. et al. Body size and risk for colorectal cancers showing BRAF mutations or microsatellite instability: a pooled analysis. Int. J. Epidemiol. 41 1060-1072 (2012).

59. Kuchiba, A. et al. Body mass index and risk of colorectal cancer according to fatty acid synthase expression in the nurses' health study. J. Natl Cancer Inst. 104, 415-420 (2012).

60. Morikawa, T. et al. Prospective analysis of body mass index, physical activity, and colorectal cancer risk associated with $\beta$-catenin (CTNNB1) status. Cancer Res. 73, 1600-1610 (2013)

61. Khandekar, M. J., Cohen, P. \& Spiegelman, B. M Molecular mechanisms of cancer development in obesity. Nat. Rev. Cancer 11, 886-895 (2011).
62. Roberts, D. L., Dive, C. \& Renehan, A. G. Biological mechanisms linking obesity and cancer risk: new perspectives. Annu. Rev. Med. 61, 301-316 (2010).

63. van Kruijsdijk, R. C., van der Wall, E. \& Visseren, F. L. Obesity and cancer: the role of dysfunctional adipose tissue. Cancer Epidemiol. Biomarkers Prev. 18 2569-2578 (2009).

64. Renehan, A. G. in Clinical Endocrine Oncology (eds Hay, I. D. \& Wass, J. A. H.) 32-40 (Oxford Univ. Press, 2008).

65. Travis, R. C. \& Key, T. J. Oestrogen exposure and breast cancer risk. Breast Cancer Res. 5, 239-247 (2003).

66. Key, T. J. et al. Body mass index, serum sex hormones, and breast cancer risk in postmenopausal women. J. Natl Cancer Inst. 95, 1218-1226 (2003). A large pooled analysis that indicates that the association between elevated BMI and postmenopausal breast cancer is, in part, mediated through circulating sex hormones.

67. Kaaks, R. et al. Postmenopausal serum androgens, oestrogens and breast cancer risk: the European prospective investigation into cancer and nutrition. Endocr. Relat. Cancer. 12, 1071-1082 (2005). A large-scale European study confirming that the association between elevated BMI and postmenopausal breast cancer is, in part, mediated through circulating sex hormones.

68. Derby, C. A., Zilber, S., Brambilla, D., Morales, K. H. \& McKinlay, J. B. Body mass index, waist circumference and waist to hip ratio and change in sex steroid hormones: the Massachusetts Male Ageing Study. Clin. Endocrinol. 65, 125-131 (2006).

69. Kaaks, R., Lukanova, A. \& Kurzer, M. S. Obesity, endogenous hormones, and endometrial cancer risk: synthetic review. Cancer Epidemiol. Biomarkers Prev. 11, 1531-1543 (2002)

70. Giovannucci, E. \& Michaud, D. The role of obesity and related metabolic disturbances in cancers of the colon, prostate, and pancreas. Gastroenterology 132 2208-2225 (2007).

71. Thompson, I. M. et al. The influence of finasteride on the development of prostate cancer. N. Engl. J. Med. 349, 215-224 (2003).

72. Platz, E. A. et al. Sex steroid hormones and the androgen receptor gene CAG repeat and subsequent risk of prostate cancer in the prostate-specific antigen era. Cancer Epidemiol. Biomarkers Prev. 14, 1262-1269 (2005).

73. Severi, G. et al. Circulating steroid hormones and the risk of prostate cancer. Cancer Epidemiol. Biomarkers Prev. 15, 86-91 (2006).

74. Vona-Davis, L. \& Rose, D. P. Adipokines as endocrine, paracrine, and autocrine factors in breast cancer risk and progression. Endocr. Relat. Cancer 14, 189-206 (2007).

75. Dirat, B., Bochet, L., Escourrou, G., Valet, P. \& Muller, C. Unraveling the obesity and breast cancer links: a role for cancer-associated adipocytes? Endocr Dev. 19, 45-52 (2010)

76. Muller, C. Tumour-surrounding adipocytes are active players in breast cancer progression. Ann. Endocrinol. 74, 108-110 (2013).

77. Chlebowski, R. T. et al. Estrogen plus progestin and colorectal cancer in postmenopausal women. N. Engl. J. Med. 350, 991-1004 (2004).

78. McKeown-Eyssen, G. Epidemiology of colorecta cancer revisited: are serum triglycerides and/or plasma glucose associated with risk? Cancer Epidemiol. Biomarkers Prev. 3, 687-695 (1994)

79. Giovannucci, E. Insulin and colon cancer. Cancer Causes Control 6, 164-179 (1995).

80. Pollak, M. The insulin and insulin-like growth factor receptor family in neoplasia: an update. Nat. Rev. Cancer 12, 159-169 (2012) An updated review detailing the complexities of the IGF system and its potential links with cancer development and cancer treatment.

81. Renehan, A. G. et al. Insulin-like growth factor (IGF)-I, IGF binding protein-3, and cancer risk: systematic review and meta-regression analysis. Lancet 363 1346-1353 (2004).

First overview dose-response meta-analysis evaluating the relationships between circulating IGFs and cancer risk.

82. Clayton, P. E., Banerjee, I., Murray, P. G. \& Renehan, A. G. Growth hormone, the insulin-like growth factor axis, insulin and cancer risk. Nat. Rev. Endocrinol. 7, 11-24 (2011). 
83. Xiang, A. H., Watanabe, R. M. \& Buchanan, T. A. HOMA and Matsuda indices of insulin sensitivity: poor correlation with minimal model-based estimates of insulin sensitivity in longitudinal settings. Diabetologia 57, 334-338 (2014)

84. Zhou, X. H. et al. Diabetes, prediabetes and cancer mortality. Diabetologia 53, 1867-1876 (2010).

85. LeRoith, D. Can endogenous hyperinsulinaemia explain the increased risk of cancer development and mortality in type 2 diabetes: evidence from mouse models. Diabetes Metab. Res. Rev. 26, 599-601 (2010).

86. Badrick, E. \& Renehan, A. G. Diabetes and cancer: 5 years into the recent controversy. Eur. J. Cancer $\mathbf{5 0}$, 2119-2125 (2014).

87. Bordeleau, L. et al. The association of basal insulin glargine and/or $n-3$ fatty acids with incident cancers in patients with dysglycemia. Diabetes Care 37, 1360-1366 (2014).

88. Renehan, A. G., Frystyk, J. \& Flyvbjerg, A. Obesity and cancer risk: the role of the insulin-IGF axis. Trends Endocrinol. Metab. 17, 328-336 (2006).

89. Tsilidis, K. K., Papatheodorou, S. I., Evangelou, E. \& Ioannidis, J. P. Evaluation of excess statistical significance in meta-analyses of 98 biomarker associations with cancer risk. J. Natl Cancer Inst. 104 1867-1878 (2012).

90. Harvie, M. et al. Increase in serum total IGF-I and maintenance of free IGF-I levels following intentional weight loss in pre-menopausal women at increased risk of breast cancer. Open Obes. J. [online] www. researchgate.net/publication/234101282 (2010)

91. Fischer-Posovszky, P., Wabitsch, M. \& Hochberg, Z Endocrinology of adipose tissue - an update. Horm. Metab. Res. 39, 314-321 (2007).

92. Schaffler, A., Scholmerich, J. \& Buechler, C. Mechanisms of disease: adipokines and breast cance - endocrine and paracrine mechanisms that connect adiposity and breast cancer. Nat. Clin. Pract. Endocrinol. Metab. 3, 345-354 (2007)

93. Bray, G. A. The underlying basis for obesity: relationship to cancer. J. Nutr. 132, 3451S-3455S (2002).

94. Rose, D. P., Komninou, D. \& Stephenson, G. D. Obesity, adipocytokines, and insulin resistance in breast cancer. Obes Rev. 5, 153-165 (2004).

95. Vansaun, M. N. Molecular pathways: adiponectin and leptin signaling in cancer. Clin. Cancer Res. 19 1926-1932 (2013).

96. Renehan, A. G., Roberts, D. L. \& Dive, C. Obesity and cancer: pathophysiological and biological mechanisms. Arch. Physiol. Biochem. 114, 71-83 (2008).

97. Gialamas, S. P. et al. Circulating leptin levels and risk of colorectal cancer and adenoma: a case-control study and meta-analysis. Cancer Causes Control 24, 2129-2141 (2013)

98. Fasshauer, M., Klein, J., Neumann, S., Eszlinger, M. \& Paschke, R. Hormonal regulation of adiponectin gene expression in 3T3-L1 adipocytes. Biochem. Biophys. Res. Commun. 290, 1084-1089 (2002).

99. Cnop, M. et al. Relationship of adiponectin to body fat distribution, insulin sensitivity and plasma lipoproteins: evidence for independent roles of age and sex. Diabetologia 46, 459-469 (2003).

100. Dalamaga, M., Diakopoulos, K. N. \& Mantzoros, C. S. The role of adiponectin in cancer: a review of current evidence. Endocr. Rev. 33, 547-594 (2012).

101. Hotamisligil, G. S. \& Erbay, E. Nutrient sensing and inflammation in metabolic diseases. Nat. Rev. Immunol. 8, 923-934 (2008).

102. Osborn, O. \& Olefsky, J. M. The cellular and signaling networks linking the immune system and metabolism in disease. Nat. Med. 18, 363-374 (2012).

103. Weisberg, S. P. et al. Obesity is associated with macrophage accumulation in adipose tissue. J. Clin Invest. 112, 1796-1808 (2003)

104. Erlinger, T. P., Platz, E. A., Rifai, N. \& Helzlsouer, K. J. $\mathrm{C}$-reactive protein and the risk of incident colorectal cancer. JAMA 291, 585-590 (2004).

105. Tsilidis, K. K. et al. C-reactive protein and colorectal cancer risk: a systematic review of prospective studies. Int. J. Cancer 123, 1133-1140 (2008).

106. Chan, A. T., Ogino, S., Giovannucci, E. L. \& Fuchs, C. S. Inflammatory markers are associated with risk of colorectal cancer and chemopreventive response to anti-inflammatory drugs. Gastroenterology 140 , 799-808 (2011)
107. Aleksandrova, K. et al. Circulating C-reactive protein concentrations and risks of colon and rectal cancer: nested case-control study within the European Prospective Investigation into Cancer and Nutrition. Am. J. Epidemiol. 172, 407-418 (2010).

108. Song, M. et al. A prospective study of plasma inflammatory markers and risk of colorectal cancer in men. Br. J. Cancer 108, 1891-1898 (2013).

109. Dieudonne, M. N. et al. Adiponectin mediates antiproliferative and apoptotic responses in human MCF7 breast cancer cells. Biochem. Biophys. Res. Commun. 345, 271-279 (2006).

110. Hursting, S. D., Nunez, N. P., Varticovski, L. \& Vinson, C. The obesity-cancer link: lessons learned from a fatless mouse. Cancer Res. 67, 2391-2393 (2007).

111. Brenner, D. R. et al. A review of the application of inflammatory biomarkers in epidemiologic cancer research. Cancer Epidemiol. Biomarkers Prev. 23, 1729-1751 (2014).

112. Byers, T. \& Sedjo, R. L. Does intentional weight loss reduce cancer risk? Diabetes Obes. Metab. 13 1063-1072 (2011).

\section{A comprehensive review of the effects of} weight-losing interventions on cancer-related intermediary biomarkers. Importantly, the review ranks the relative biomarker changes per $10 \%$ weight reduction.

113. Mason, C. et al. Effects of dietary weight loss and exercise on insulin-like growth factor-I and insulin-like growth factor-binding protein-3 in postmenopausal women: a randomized controlled trial. Cancer Epidemiol. Biomarkers Prev. 22, 1457-1463 (2013).

114. Balagopal, P. B. et al. Nontraditional risk factors and biomarkers for cardiovascular disease: mechanistic, research, and clinical considerations for youth: a scientific statement from the American Heart Association. Circulation 123, 2749-2769 (2011).

115. Canoy, D. et al. Body fat distribution and risk of coronary heart disease in men and women in the European Prospective Investigation Into Cancer and Nutrition in Norfolk cohort: a population-based prospective study. Circulation 116, 2933-2943 (2007).

One of only a few large-scale studies to evaluate the associations between hip and gluteal fat distribution and risk of a common metabolic disease, coronary heart disease.

116. Stefan, N., Haring, H. U., Hu, F. B. \& Schulze, M. B. Metabolically healthy obesity: epidemiology, mechanisms, and clinical implications. Lancet Diabetes Endocrinol. 1, 152-162 (2013)

117. Moore, L. L., Chadid, S., Singer, M. R., Kreger, B. E. \& Denis, G. V. Metabolic health reduces risk of obesityrelated cancer in framingham study adults. Cancer Epidemiol. Biomarkers Prev. 23, 2057-2065 (2014)

118. Britton, K. A. \& Fox, C. S. Ectopic fat depots and cardiovascular disease. Circulation 124, e837-841 (2011).

A seminal overview introducing the concepts of ectopic fat and metabolic disease predisposition.

119. Cornier, M. A. et al. Assessing adiposity: a scientific statement from the American Heart Association. Circulation 124, 1996-2019 (2011).

120. Ouchi, N., Parker, J. L., Lugus, J. J. \& Walsh, K. Adipokines in inflammation and metabolic disease. Nat. Rev. Immunol. 11, 85-97 (2011)

121. Fabbrini, E. et al. Intrahepatic fat, not visceral fat, is linked with metabolic complications of obesity. Proc Natl Acad. Sci. USA 106, 15430-15435 (2009).

122. Kahn, B. B. \& Flier, J. S. Obesity and insulin resistance J. Clin. Invest. 106, 473-481 (2000).

123. Aleksandrova, K., Nimptsch, K. \& Pischon, T. Obesity and colorectal cancer. Front. Biosci. (Elite Ed.) 5, 61-77 (2013)

124. Moore, L. L. et al. BMI and waist circumference as predictors of lifetime colon cancer risk in Framingham Study adults. Int. J. Obes. Relat. Metab. Disord. 28, 559-567 (2004)

125. Pischon, T. et al. Body size and risk of colon and rectal cancer in the European Prospective Investigation Into Cancer and Nutrition (EPIC). J. Natl Cancer Inst. 98, 920-931 (2006).

One of the first large-scale studies to suggest that indices of central adiposity, such as waist circumference, may be more informative than BMI in the prediction of cancer risk.

126. Folsom, A. R. et al. Associations of general and abdominal obesity with multiple health outcomes in older women: the lowa Women's Health Study. Arch. Intern. Med. 160, 2117-2128 (2000).
127. Keimling, M. et al. Comparison of associations of body mass index, abdominal adiposity, and risk of colorectal cancer in a large prospective cohort study. Cancer Epidemiol. Biomarkers Prev. 22, 1383-1394 (2013).

A large study directly comparing the associations of waist circumference versus BMI and colorectal cancer risk, and challenging the notion that there are differences in the strengths of these different associations.

128. Oxentenko, A. S. et al. Body size and incident colorectal cancer: a prospective study of older women. Cancer Prev. Res. 3, 1608-1620 (2010).

129. Gaudet, M. M. et al. Waist circumference, body mass index, and postmenopausal breast cancer incidence in the Cancer Prevention Study-II Nutrition Cohort. Cancer Causes Control 25, 737-745 (2014).

130. Huang, Z. et al. Waist circumference, waist:hip ratio, and risk of breast cancer in the Nurses' Health Study. Am. J. Epidemiol. 150, 1316-1324 (1999).

131. World Cancer Research Fund and American Institute for Cancer Research. Continuous Update Project Report. Food, Nutrition, Physical Activity, and the Prevention of Pancreatic Cancer. (AICR, 2012).

132. World Cancer Research Fund and American Institute for Cancer Research. Continuous Update Project Report. Food, Nutrition, Physical Activity, and the Prevention of Endometrial Cancer. (AICR, 2013).

133. Greenstein, A. S. et al. Local inflammation and hypoxia abolish the protective anticontractile properties of perivascular fat in obese patients. Circulation 119, 1661-1670 (2009).

134. Fox, C. S. et al. Periaortic fat deposition is associated with peripheral arterial disease: the Framingham heart study. Circ. Cardiovasc. Imag. 3, 515-519 (2010).

135. Schautz, B., Later, W., Heller, M., Muller, M. J. \& Bosy-Westphal, A. Associations between breast adipose tissue, body fat distribution and cardiometabolic risk in women: cross-sectional data and weight-loss intervention. Eur. J. Clin. Nutr. 65, 784-790 (2011)

136. Martinez-Outschoorn, U. E., Sotgia, F. \& Lisanti, M. P. Caveolae and signalling in cancer. Nat. Rev. Cancer 15, 225-237 (2015)

137. Park, J., Morley, T. S., Kim, M., Clegg, D. J. \& Scherer, P. E. Obesity and cancer - mechanisms underlying tumour progression and recurrence. Nat. Rev. Endocrinol. 10, 455-465 (2014).

138. Stienstra, R. et al. Inflammasome is a central player in the induction of obesity and insulin resistance. Proc. Natl Acad. Sci. USA 108, 15324-15329 (2011).

139. Stefan, N., Kantartzis, K. \& Haring, H. U. Causes and metabolic consequences of fatty liver. Endocr. Rev. 29 939-960 (2008).

A comprehensive review detailing the importance of fat deposition in the liver as a determinant of insulin resistance.

140. Tacke, F., Luedde, T. \& Trautwein, C. Inflammatory pathways in liver homeostasis and liver injury. Clin. Rev. Allergy Immunol. 36, 4-12 (2009).

141. Park, E. J. et al. Dietary and genetic obesity promote liver inflammation and tumorigenesis by enhancing IL-6 and TNF expression. Cell 140, 197-208 (2010). A seminal biological paper of a diet-induced hepatocellular carcinoma model in mice, identifying the key role of inflammatory mediators.

142. Wang, Y., Ausman, L. M., Greenberg, A. S. Russell, R. M. \& Wang, X. D. Nonalcoholic steatohepatitis induced by a high-fat diet promotes diethylnitrosamine-initiated early hepatocarcinogenesis in rats. Int. J. Cancer $\mathbf{1 2 4}$, 540-546 (2009)

143. Michelotti, G. A., Machado, M. V. \& Diehl, A. M. NAFLD, NASH and liver cancer. Nat. Rev. Gastroenterol. Hepatol. 10, 656-665 (2013).

144. Hart, C. L., Morrison, D. S., Batty, G. D., Mitchell, R. J. $\&$ Davey Smith, G. Effect of body mass index and alcohol consumption on liver disease: analysis of data from two prospective cohort studies. BMJ 340, c1240 (2010).

A key paper demonstrating the complex interactions between risk factors for liver disease and mortality, including cancer-related mortality.

145. Sijens, P. E., Edens, M. A., Bakker, S. J. \& Stolk, R. P. MRI-determined fat content of human liver, pancreas and kidney. World J. Gastroenterol. 16, 1993-1998 (2010).

146. Lashinger, L. M. et al. Genetic reduction of insulin-like growth factor-1 mimics the anticancer effects of calorie restriction on cyclooxygenase-2-driven pancreatic neoplasia. Cancer Prev. Res. 4, 1030-1040 (2011). 
147. Hori, M. et al. Association of pancreatic Fatty infiltration with pancreatic ductal adenocarcinoma. Clin. Transl. Gastroenterol. 5, e53 (2014).

148. Bertolini, F., Petit, J. Y. \& Kolonin, M. G. Stem cells from adipose tissue and breast cancer: hype, risks and hope. Br. J. Cancer 112, 419-423 (2015).

A review of innovative thinking on the interplay between stem cells, adipose tissue and cancer development.

149. Tremaroli, V. \& Backhed, F. Functional interactions between the gut microbiota and host metabolism. Nature 489, 242-249 (2012)

150. Schulz, M. D. et al. High-fat-diet-mediated dysbiosis promotes intestinal carcinogenesis independently of obesity. Nature 514, 508-512 (2014).

151. Ohtani, N. Microbiome and cancer. Semin Immunopathol. 37, 65-72 (2015).

152. Thanassoulis, G. et al. Prevalence, distribution, and risk factor correlates of high pericardial and intrathoracic fat depots in the Framingham heart study. Circ. Cardiovasc. Imag. 3, 559-566 (2010).

153. Hernan, M. A., Hernandez-Diaz, S. \& Robins, J. M. A structural approach to selection bias. Epidemiology 15, 615-625 (2004)

A key paper detailing the ways in which epidemiological studies can infer causality.

154. Hernan, M. A. \& Robins, J. M. Instruments for causal inference: an epidemiologist's dream? Epidemiology 17, 360-372 (2006)

155. Smith, G. D. \& Ebrahim, S. Mendelian randomization: prospects, potentials, and limitations. Int. J. Epidemiol. 33, 30-42 (2004).
156. Smith, G. D., Timpson, N. \& Ebrahim, S. Strengthening causal inference in cardiovascular epidemiology through Mendelian randomization. Ann. Med. 40, 524-541 (2008)

157. Kilpelainen, T. O. et al. Genetic variation near IRS associates with reduced adiposity and an impaired metabolic profile. Nat. Genet. 43, 753-760 (2011).

158. Speliotes, E. K. et al. Association analyses of 249,796 individuals reveal 18 new loci associated with body mass index. Nat. Genet. 42, 937-948 (2010).

159. Tao, M. H. et al. Polymorphisms in the CYP19A (aromatase) gene and endometrial cancer risk in Chinese women. Cancer Epidemiol. Biomarkers Prev. 16, 943-949 (2007).

160. Wen, W. et al. The modifying effect of C-reactive protein gene polymorphisms on the association between central obesity and endometrial cancer risk. Cancer 112, 2409-2416 (2008).

161. Lurie, G. et al. The obesity-associated polymorphisms FTO rs9939609 and MC4R rs 17782313 and endometrial cancer risk in non-Hispanic white women. PLOS ONE 6, e 16756 (2011).

162. Lewis, S. J. et al. Associations between an obesity related genetic variant (FTO rs9939609) and prostate cancer risk. PLoS ONE 5, e13485 (2010)

163. Tenesa, A. et al. Common genetic variants at the $M C 4 R$ locus are associated with obesity, but not with dietary energy intake or colorectal cancer in the Scottish population. Int. J. Obes. 33, 284-288 (2009).

164. Sainz, J. et al. GWAS-identified common variants for obesity are not associated with the risk of developing colorectal cancer. Cancer Epidemiol. Biomarkers Prev. 23, 1125-1128 (2014).
165. Bellamy, S. L, Lin, J Y \& Ten Have, T. R. An introduction to causal modeling in clinical trials. Clin Trials 4, 58-73 (2007).

166. Dossus, L. et al. Hormonal, metabolic, and inflammatory profiles and endometrial cancer risk within the EPIC Cohort - a factor analysis. Am J. Epidemiol. 177, 787-799 (2013). A multi-compartment analysis exploring new aetiological pathways linking adiposity and cancer risk. Notably, the study ranks the relative importance of the tested pathways.

167. Nead, K. T. et al. Evidence of a causal association between insulinemia and endometrial cancer: a Mendelian randomization analysis. J. Natl Cancer Inst. 107, djv178 (2015).

Acknowledgements

The authors acknowledge the many colleagues and othe researchers around the world who have shared with them, over many years, their thoughts, experiences and methodological insights into the details and concepts described in this Review.

\section{Competing interests statement}

The authors declare no competing interests. 